\title{
Partial Inactivation of the Primary Motor Cortex Hand Area: Effects on Individuated Finger Movements
}

\author{
Marc H. Schieber ${ }^{1,2,3,4}$ and Andrew V. Poliakov ${ }^{1}$ \\ Departments of ${ }^{1}$ Neurology and ${ }^{2}$ Neurobiology and Anatomy, Brain and Cognitive Science, and Physical Medicine and \\ Rehabilitation, ${ }^{3}$ Center for Visual Science, and ${ }^{4 B}$ Brain Injury Rehabilitation Program at St. Mary's Hospital, University of \\ Rochester School of Medicine and Dentistry, Rochester, New York 14642
}

\begin{abstract}
After large lesions of the primary motor cortex (M1), voluntary movements of affected body parts are weak and slow. In addition, the relative independence of moving one body part without others is lost; attempts at individuated movements of a given body part are accompanied by excessive, unintended motion of contiguous body parts. The effects of partial inactivation of the $\mathrm{M} 1$ hand area are comparatively unknown, however. If the M1 hand area contains the somatotopically ordered finger representations implied by the classic homunculus or simiusculus, then partial inactivation might produce weakness, slowness, and loss of independence of one or two adjacent digits without affecting other digits. But if control of each finger movement is distributed in the M1 hand area as many studies suggest, then partial inactivation might produce dissociation of weakness, slowness, and relative independence of movement, and which fingers movements are impaired might be unrelated to the location of the inactivation along the central sulcus.
\end{abstract}

To investigate the motoric deficits resulting from partial inactivation of the M1 hand area, we therefore made single intracortical injections of muscimol as trained monkeys performed visually cued, individuated flexion-extension movements of the fingers and wrist. We found little if any evidence that which finger movements were impaired after each injection was related to the injection location along the central sulcus. Unimpaired fingers could be flanked on both sides by impaired fingers, and the flexion movements of a given finger could be unaffected even though the extension movements were impaired, or vice versa. Partial inactivation also could produce dissociated weakness and slowness versus loss of independence in a given finger movement. These findings suggest that control of each individuated finger movement is distributed widely in the M1 hand area.

Key words: cortex; dexterity; finger; individuation; macaque; motor; muscimol; response time, somatotopy; weakness
The classic homunculus and simiusculus implied that a separate region of the primary motor cortex (M1) moves each digit of the hand (Penfield and Rasmussen, 1950; Woolsey et al., 1951). Evidence now suggests, however, that control of any finger movement is distributed throughout the M1 hand area (see Discussion), calling into question the degree to which a somatotopic map of the fingers mediates the crucial contribution of M1 to performance of individuated finger movements. Clearly, lesions of lateral M1 impair voluntary movement of the face much more than the leg and vice versa for medial M1 lesions. If such somatotopic organization extends to the level of different fingers, then lateral lesions within the hand area should impair thumb movements more than little finger movements and vice versa for medial lesions. We therefore sought to reversibly inactivate only part of the M1 hand area, because such within-hand somatotopic effects would not have been evident in previous studies of lesions in M1 or the corticospinal tract. These studies either ablated the entire M1 hand area (Fulton and Kennard, 1932; Passingham et al., 1983) or eliminated most of the corticospinal outflow by cutting the pyramidal tract (Tower, 1940; Lawrence and Kuypers, 1968; Hepp-Reymond et al., 1970;

Received March 2, 1998; revised Aug. 10, 1998; accepted Aug. 13, 1998.

This work was supported by Grant R01-NS27686 from the National Institute of Neurological Disorders and Stroke. We thank W. Thomas Thach Jr. for suggesting these studies, Jennifer Gardinier for technical assistance, and Marsha Hayles for editorial comments.

Correspondence should be addressed to Dr. Marc H. Schieber, University of Rochester Medical Center, Department of Neurology, 601 Elmwood Avenue, Box 673, Rochester, NY 14642.

Copyright (C) 1998 Society for Neuroscience $\quad 0270-6474 / 98 / 189038-17 \$ 05.00 / 0$
Woolsey et al., 1972), rather than selectively ablating only part of the hand area. Moreover, functional recovery after permanent lesions-either cortical (Nudo and Milliken, 1996; Nudo et al., 1996) or pyramidal (Schwartzman, 1978; Chapman and Wiesendanger 1982)—would have provided an opportunity for plastic changes to mask prelesion somatotopic organization.

Intracortical injection of muscimol, a long-acting $\mathrm{GABA}_{\mathrm{A}}$ agonist, recently has been shown to produce reversible inactivation of the M1 hand area, with associated deficits in motor performance (Kubota, 1996; Rouiller et al., 1997). These studies to date have used injections of relatively large amounts of muscimol at multiple sites, intended to maximize the motor deficit. Yet single injections of small amounts in cat M1 have been found to produce measurable deficits in reaching behavior (Martin and Ghez, 1993; Martin et al., 1993). Small amounts of muscimol injected into the primate M1 hand area therefore might produce a localized inactivation that could be used to further investigate the degree of within-hand somatotopy. Even if muscimol eventually spread throughout the M1 hand area, somatotopic organization should be observable as the muscimol diffused. For example, laterally placed injections would be expected to impair thumb movements first, whereas medially placed injections should first affect the little finger.

We therefore made single intracortical injections of muscimol in the M1 hand area as monkeys performed individuated finger movements. These injections impaired performance of some finger movements, whereas other finger movements remained unaffected. Which finger movements were impaired after each muscimol injection showed little if any relation to the location of the 
injection along the central sulcus, however, consistent with distributed control of each finger movement.

\section{MATERIALS AND METHODS}

All procedures for the care and use of these purpose-bred monkeys complied with the United States Public Health Service Policy on Humane Care and Use of Laboratory Animals, followed the Public Health Service Guide for the Care and Use of Laboratory Animals and were approved by the appropriate Institutional Animal Care and Use Committee.

Visually cued individuated finger movement task. Three juvenile ( $\sim 4-$ to 6-yr-old) rhesus monkeys (Macaca mulatta; K, a $6 \mathrm{~kg}$ male; H, a $4 \mathrm{~kg}$ female; and $\mathrm{A}$, a $5 \mathrm{~kg}$ male) were trained to perform visually cued individuated finger movements. The behavioral paradigm and the finger movements made by monkey $\mathrm{K}$ have been described in detail previously (Schieber, 1991). The monkey's elbow was restrained in a molded cast, and the right hand was placed in a pistol-grip manipulandum that separated each finger into a different slot. At the end of each slot, each fingertip lay between two microswitches. By flexing or extending a digit a few millimeters, the monkey closed the ventral or dorsal switch, respectively. This pistol-grip manipulandum was mounted, in turn, on an axis permitting flexion and extension wrist movements. A potentiometer coupled to the axis transduced wrist motion, and the output of this potentiometer was fed to level-crossing circuits that simulated flexion and extension switches for the wrist.

The monkey viewed a display on which each digit (and the wrist) was represented by a row of five light-emitting diodes (LEDs). The middle, yellow LED in a row was illuminated when neither the flexion nor extension switch for that digit was closed. One of two green LEDs on either side of the middle, yellow LED was lit whenever the flexion (leftward green LED) or extension (rightward green LED) switch was closed. When the monkey flexed or extended a digit, closing a microswitch, the middle, yellow LED went out and the leftward or rightward green LED came on. The yellow and green LEDs thus informed the monkey which switches were open and which were closed. Red LEDs at either end of the row were illuminated as cues instructing the monkey to close either the flexion (leftward red LED) or extension (rightward red LED) switch.

The monkey initiated each trial by placing all digits and the wrist in their middle positions, so that no switches were closed and the middle, yellow LED in each row was illuminated. After a pseudorandomly varied initial hold period of $500-750 \mathrm{msec}$, one red LED was illuminated under microprocessor control, instructing the monkey which switch to close (or to move the wrist). If the monkey closed the instructed switch within the $700 \mathrm{msec}$ allowed response time after illumination of the red LED and held it closed for a final hold period ( $500 \mathrm{msec}$ for monkeys $\mathrm{K}$ and $\mathrm{H}, 300$ msec for monkey A) without closing any other switches, then the trial had been performed correctly, and the monkey received a water reward. After each rewarded trial, the finger movement to be instructed for the next trial was rotated in a pseudorandom order. Consecutive rewarded (correctly performed) trials of a given instructed movement therefore did not occur immediately after one another but instead were separated by trials of other instructed movements. In contrast, if the monkey failed to perform correctly, either by failing to close the instructed switch within the allowed $700 \mathrm{msec}$ response time or by closing another switch before or after the instructed switch, no reward was delivered, and the same instruction was presented again for the next trial. Consecutive failed trials of a given instructed movement therefore did occur immediately after one another and were not separated by trials of other instructed movements. (This protocol was required to prevent the monkey from intentionally passing up trials of difficult movements by failing them and then going on to earn rewards only on trials of easier movements.) After each trial, a minimum intertrial interval (1000 msec for monkeys $\mathrm{K}$ and $\mathrm{H}, 500 \mathrm{msec}$ for monkey A) was required before the monkey could initiate the next trial. Because the monkey had to initiate each trial by actively placing all digits and the wrist in their middle positions, the actual intertrial interval was variable, determined in part by the monkey.

Although the behavioral task was based only on switch closures, in recording from monkeys $\mathrm{K}$ and $\mathrm{H}$ the position of each finger was transduced continuously via strain gages mounted on the microswitch lever arms, and the position of the wrist was transduced via a potentiometer coupled to the wrist axis (Schieber, 1991). These signals were sampled at $100 \mathrm{~Hz}$ and stored in data files along with codes marking the times of behavioral events in each movement trial. In monkeys $\mathrm{K}$ and $\mathrm{H}$, data were collected and stored only during trials and not during intertrial intervals, precluding our constructing an accurate time base across multiple trials. In describing the results, we therefore show the temporal sequence of changes in a given session as a function of trial number in that session. Nevertheless, from handwritten notes we were able to obtain typical values of the total elapsed time in some sessions and of the time required for performing the injection in these sessions.

Examination of the finger movements generated by these monkeys showed that in each rewarded trial, the digit the monkey had been instructed to move underwent more movement than any other digit. Moreover, each digit had its greatest excursion when it was the instructed digit. In some movements, particularly when the monkey was instructed to flex the thumb or wrist, other digits remained stationary. In other movements, however, noninstructed digits moved to a greater or lesser degree. Each movement is therefore referred to as an instructed movement of a given digit in a given direction, recognizing that there was often some movement of noninstructed digits. For brevity, each instructed movement is denoted by the number of the instructed digit ( 1 for the thumb through 5 for the little finger, W for the wrist) and the first letter of the instructed direction ( $\mathrm{f}$ for flexion, e for extension). Thus " $\mathrm{f}$ " denotes instructed flexion of the index finger. Monkeys $\mathrm{K}$ and $\mathrm{H}$ were trained to perform 12 or more different finger and wrist movements, although for purposes of exposition only individuated flexion and extension of each digit and of the wrist are analyzed here. Monkey A, in contrast, was trained to perform only six movements, $1 \mathrm{f}, 2 \mathrm{f}, 3 \mathrm{f}, 4 \mathrm{f}, 2 \mathrm{e}$, and $3 e$, all with the wrist axis fixed.

Quantifying the independence of the digits. To quantify the degree to which noninstructed digits moved simultaneously with the instructed digit in single trials of each instructed movement, we applied methods previously described in detail (Schieber, 1991). Because the motion of noninstructed digits tended to parallel the motion of the instructed digit in a given trial, plots of the position of a noninstructed digit as a function of the simultaneous position of the instructed digit typically had a major linear component (see Fig. 6A,B). For each such plot, the slope of the best-fit line therefore was used as a coefficient quantifying the relative motion of the noninstructed digit during movement of the instructed digit. This coefficient was close to 0 if the noninstructed digit remained stationary as the instructed digit moved but was closer to 1 the more the noninstructed digit moved along with the instructed digit and was negative if the noninstructed digit moved in the direction opposite the movement of the instructed digit.

To summarize the extent to which noninstructed digits moved during a given instructed movement, we then computed an Individuation Index as 1 - mean of the absolute values of the relative motion coefficients of the noninstructed digits:

$$
\mathrm{II}_{j d}=1-\left[\left(\sum_{i=1}^{n}\left|S_{i j d}\right|\right)-1\right] /(n-1),
$$

where $\mathrm{II}_{j d}$ is the Individuation Index for instructed movement of the $j$ th digit in direction $d$ (flexion or extension); $S_{i j d}$ is the relative motion coefficient of the $i$ th digit during instructed movement of the $j$ th digit in direction $d$; and $n$ is the number of digits (here $n=6$ because the wrist is included). The slope of the instructed digit against itself, which is always 1 , was eliminated from the sum by subtracting 1 in the numerator, and the number of noninstructed digits $(n-1)$ was used as the denominator. If the noninstructed digits remained stationary during the instructed movement, the Individuation Index was close to 1 . The more the noninstructed digits moved, however, the closer the Individuation Index was to 0 .

Natural food retrieval task. In addition to these highly overtrained movements, all three monkeys were examined and videotaped retrieving small food morsels (apple pieces $\sim 0.5-1 \mathrm{cc}$ ) from two wells machined in a block of clear Lucite: one well large enough to permit entry of the entire hand ( $89 \mathrm{~mm}$ high, $38 \mathrm{~mm}$ wide, and $45 \mathrm{~mm}$ deep) and another well small enough to permit entry of only one finger $(29 \mathrm{~mm}$ high, $8 \mathrm{~mm}$ wide, and $23 \mathrm{~mm}$ deep). An investigator presented these food wells to the monkey one at a time while the monkey sat on the floor (monkey K) in a cage with two openings large enough to permit the hand and forearm to reach through freely on the monkey's right and left (monkey $\mathrm{H}$ ) or in the primate chair restrained only by the collar (monkey A). The wells were presented at eye level (monkeys $\mathrm{K}$ and $\mathrm{A}$ ) or at floor level (monkey $\mathrm{H})$. To induce the monkey to use the right hand on some trials and the left hand on other trials, the food wells were presented at a comfortable reaching distance to the monkey's right or left, respectively. 
Identification of the M1 hand area. Before any muscimol injection sessions, the M1 hand area was identified physiologically in each monkey. Conventional microelectrode techniques were used to record single neuron activity as the monkey performed the visually cued, individuated finger movement task. As previously reported (Schieber and Hibbard, 1993), single neurons active in relation to any given finger movement were found over a region of M1 extending 6-9 $\mathrm{mm}$ along the anterior bank and lip of the central sulcus. This territory was considered the M1 hand region. This was confirmed by switching the connections of the microelectrode from the recording preamplifier to a stimulus isolator (BAK BSI-1) and delivering conventional intracortical microstimulation (ICMS; trains of 12, biphasic, $200 \mu \mathrm{sec}, 5-40 \mu \mathrm{A}$ constant current pulses at $330 \mathrm{~Hz}$ ) as the awake monkey performed the visually cued, individuated finger movement task. ICMS was triggered under computer control as the monkey waited in the initial hold period of the task for an instruction cue or by the investigator as the monkey rested quietly between trials. Responses to ICMS were identified in monkeys $\mathrm{K}$ and $\mathrm{H}$ by observing evoked movements of the fingers or wrist and by palpating contractions of forearm and upper arm muscles. In monkey A, ICMS responses were identified in averages of rectified EMG recorded through percutaneously implanted electrodes (Mewes and Cheney, 1991). In each of the three monkeys, the cortical territory from which ICMS evoked visible finger movements or EMG responses was coextensive with the region containing task-related neurons.

Experimental sessions. Reversible inactivation of M1 was produced by intracortical injection of the $\mathrm{GABA}_{\mathrm{A}}$ agonist muscimol (Sigma, St. Louis, MO). Muscimol was prepared for injection in physiological PBS, $\mathrm{pH} 7.4$, at a concentration of $5 \mu \mathrm{g} / \mu \mathrm{l}$ (for a few injections, concentrations of 1 or $10 \mu \mathrm{g} / \mu \mathrm{l}$ were used), sterilized by passage through a micropore filter, and kept refrigerated until use. In experimental sessions, the monkey first was videotaped as he retrieved food morsels from the large and small food wells. The monkey then was placed in the primate chair, the recording chamber was opened, and a microelectrode was lowered into the brain at coordinates chosen from previous neuron recording sessions, confirming the depth of the cortical gray matter on the anterior lip and in the anterior bank of the central sulcus. After withdrawing the microelectrode, a $1 \mu$ l syringe (Unimetrics, Chicago, IL) loaded with solution for injection was mounted on the same micropositioner, such that its 30 gauge needle passed along the same line as the microelectrode. The needle then was passed through the dural puncture left by the microelectrode and advanced into the brain to the depth of cortex defined from the immediately preceding microelectrode recording. Once the needle was in place, the monkey began performing visually cued, individuated finger movements. After baseline finger movement data had been collected, the muscimol solution was injected by hand in aliquots of $0.1 \mu$ l every $30 \mathrm{sec}$ over $5 \mathrm{~min}$. The needle then was left in place while the monkey continued performing the finger movement task as further performance data were collected. In two M1 sessions (31 and 32 in monkey $\mathrm{H}$ ), however, the needle was removed after the first injection and reloaded with muscimol, and a second injection was made $3 \mathrm{~mm}$ deeper than the first. In premotor cortex (PM) sessions, six to nine total injections were made in three different penetration tracks.

Once the monkey had stopped performing the visually cued, individuated movement task, either because of muscimol-induced inability or because of satiation, the needle was withdrawn, and the recording chamber was cleaned and closed. The monkey's hand was removed from the pistol-grip manipulandum and examined clinically for posture, tone, and strength. Then the monkey's postinjection behavior retrieving food morsels from the large and small food wells was evaluated and recorded on videotape. Thereafter, the monkey was returned to its home cage for the night.

Experimental sessions were always separated from one another by at least $1 \mathrm{~d}$. The day after most muscimol injections, the monkey was again videotaped retrieving food morsels from the food wells and again worked at the visually cued, individuated finger movement task. The day after each muscimol injection, performance of both behaviors had returned to baseline levels.

Histology. After the completion of all experiments on each monkey, electrolytic lesions were made by passing DC current (40 $\mu \mathrm{A}$ for $40 \mathrm{sec}$ ) through a microelectrode at selected locations. Several days later, the monkey was tranquilized with ketamine $(10 \mathrm{mg} / \mathrm{kg}, \mathrm{i} . \mathrm{m}$.$) , killed by lethal$ injection of thiopental $(300 \mathrm{mg} / \mathrm{kg}$, i.v.), and perfused transcardially with PBS followed by phosphate-buffered $4 \%$ paraformaldehyde. Before removing the brain from the cranium and photographing the cortical surfaces, marking ink tracks were placed at selected locations around the muscimol injection sites via a needle mounted on the same microdrive. Frozen sections of the frontal lobes of both hemispheres were cut in the coronal plane at $30 \mu \mathrm{m}$, and every fourth section was mounted and stained for Nissl substance. The location of each muscimol injection was reconstructed based on examination of these sections. When the track of a particular injection could not be identified in the histological material, its location was interpolated based on the coordinates at which the injection was made and the locations of identified tracks, electrolytic lesions, and postmortem inked tracks.

\section{RESULTS}

We injected muscimol to reversibly inactivate the left M1 hand area in 20 different sessions in three monkeys: $\mathrm{K}, \mathrm{H}$, and A (Table 1). In most of these sessions, $\sim 1 \mu$ l of buffered saline containing $5 \mu \mathrm{g}$ of muscimol was injected at a single site, but in two sessions muscimol was injected at two different depths along the same track, and in two other sessions the concentration of muscimol injected was 1 or $10 \mu \mathrm{g} / \mu \mathrm{l}$. To control for the mechanical effects of placing an injection needle in the M1 hand area and injecting $1 \mu$ of fluid volume, in three other sessions we made sham injections of $1 \mu \mathrm{l}$ of buffered saline in the left M1 hand area. To control for nonspecific effects that might be produced by longrange diffusion of muscimol through the cortex, CSF, or bloodstream and to control for the monkey's potential distraction caused by the investigator performing the injection, in seven other sessions we injected muscimol at distant sites: in the left M1 leg area, in the PM, or in the supplementary motor area (SMA). Many of the sessions in which injections were made outside the M1 hand area entailed injections at multiple depths and in more than one needle track, reaching much larger total doses of muscimol than used in the M1 hand area sessions.

All these sessions are summarized in Table 1, which gives the concentration of muscimol, number of sites injected, volume injected per site, total injected volume, total injected muscimol, and depth of each injection below the hemispheric surface for each session. Figure 1 shows the location of the injection sites for each session in each of the three monkeys. Session numbers in Table 1 and Figure 1 indicate the sequential order in which sessions were performed; omitted numbers represent sessions in which other cortical regions were studied, the results of which will be reported elsewhere.

The heavy dashed lines in Figure 1 indicate the surface projection of the M1 territory in each monkey where neurons were found to discharge in relation to performance of visually cued individuated finger movements and where finger movements were evoked by ICMS. M1 hand area injections were made within this territory, primarily in the lip (Table 1, depths of 2-3 mm below the hemispheric surface) and anterior bank (depths of 3-6 mm) of the central sulcus, where the digits are most heavily represented, rather than on the convexity of the precentral gyrus. Although some studies have distinguished rostral and caudal subdivisions of area 4 (Strick and Preston, 1978; Preuss et al., 1997), we did not attempt to differentiate these subdivisions physiologically or histologically, nor did we intend to inactivate them selectively. Nevertheless, because our injections were placed largely in the anterior bank and lip of the central sulcus, we are likely to have inactivated the caudal subdivision of area 4 more than the rostral subdivision.

\section{Failure of individuated finger movements after muscimol injection}

The success or failure of each individuated finger movement trial in two different sessions is illustrated in Figure 2. For each session, trials have been sorted according to the 12 instructed 


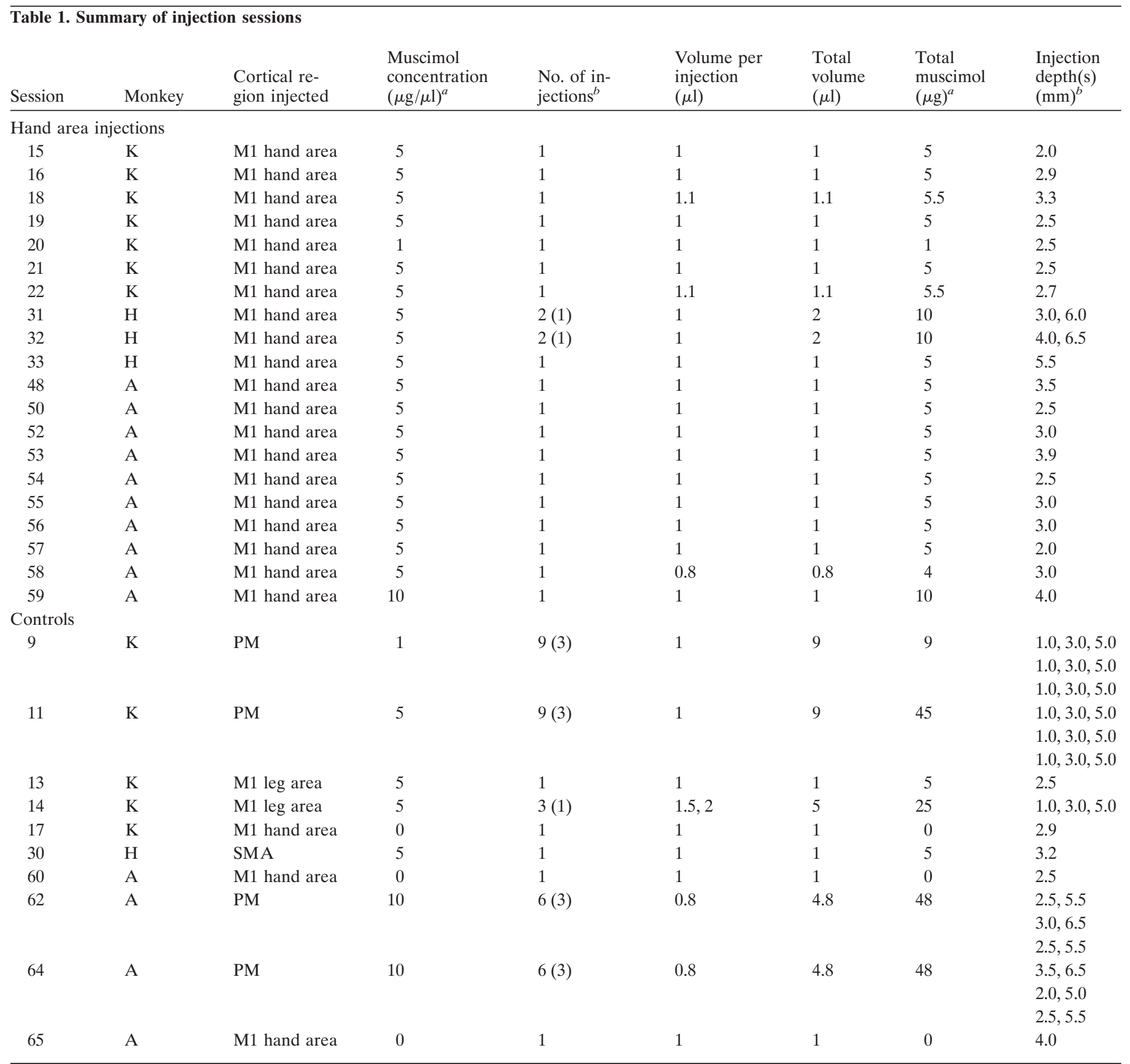

${ }^{a_{0}}$ indicates sham injection with buffered saline.

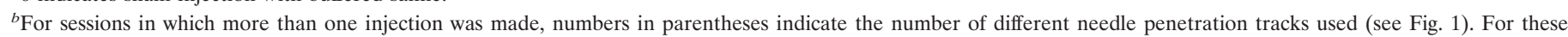
sessions, the depths at which injections were made along each needle penetration track are indicated in a separate row of the Injection depth(s) column.

movements. Successful performance on each trial is indicated by an upward tick mark, and failure is indicated by a downward tick. Before the injection in session 17, the monkey failed occasional trials, particularly of instructed movements $4 \mathrm{f}$ and $5 \mathrm{e}$. A sham injection of saline was made over $13 \mathrm{~min}$ as the monkey performed trials 334-582 (bar at bottom). After this injection the monkey's performance remained unchanged, however, and he continued to work for a total of $93 \mathrm{~min}$, performing $>2200$ trials.

Such was not the case in session 21 , during which $5 \mu \mathrm{g}$ of muscimol in $1 \mu \mathrm{l}$ of saline was injected at a single site. Before the muscimol injection, the monkey performed the majority of individuated finger movement trials successfully and failed only on occasional trials of instructed movements $4 \mathrm{f}$ and 5f. Muscimol then was injected over $5 \mathrm{~min}$ as the monkey performed trials 317-431 (Fig. 2, bar at bottom), during which his success changed only in that two failures of movement 5e occurred. Shortly after the injection was complete, the monkey failed a trial of $2 \mathrm{e}$. Although the next two trials of $2 \mathrm{e}$ were performed successfully, the monkey thereafter became unable to perform $2 \mathrm{e}$, failing repeatedly. This instructed movement therefore was removed from the rotation for several trials and subsequently was reintroduced on three occasions, but the monkey continued to fail every trial of 2e. Meanwhile, failures on trials of $5 \mathrm{e}, 5 \mathrm{f}$, and $4 \mathrm{f}$ were becoming more frequent, and eventually these movements too 
$\mathbf{K}$
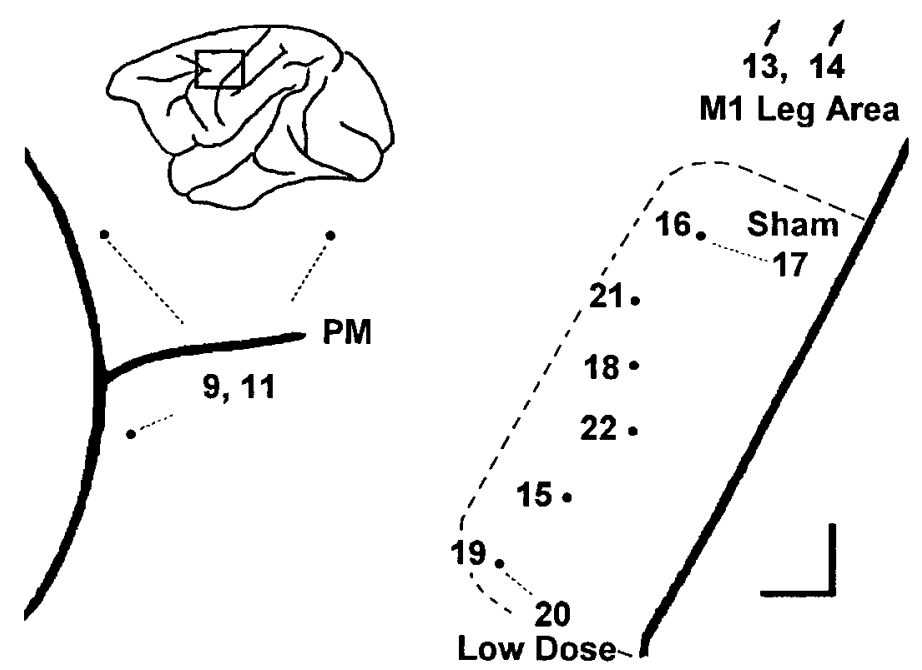

H

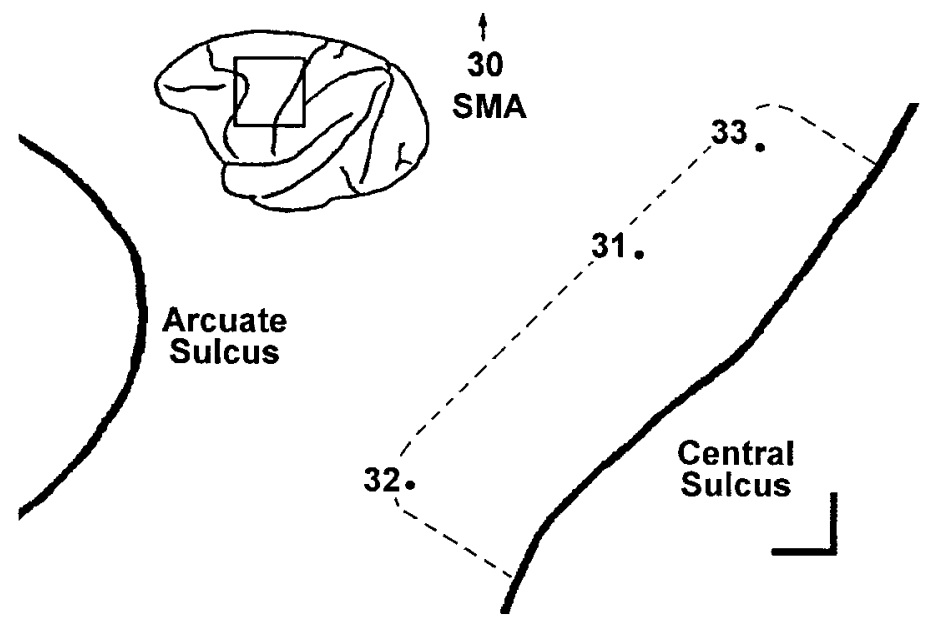

Figure 1. Location of injections in each session. For each monkey, $\mathrm{K}, \mathrm{H}$, and $\mathrm{A}$, the sites of muscimol injections are shown as points on an enlarged view of the left hemisphere, with the central sulcus to the viewer's right and the arcuate sulcus to the left. A rectangle in the inset of each monkey's left hemisphere shows the region enlarged. Session numbers are shown either next to the point representing the injection site or else connected to the point with a fine dashed line. The extent of the M1 hand area in each monkey, as assessed with single-neuron recording and ICMS, is indicated by a heavy dashed line. Scale bars at the bottom right of each enlargement indicate $1 \mathrm{~mm}$ in the rostral and medial directions. In addition to muscimol injections in the M1 hand area, comparison control injections were made in the in the PM just posterior to the arcuate sulcus, in the M1 leg area, and in the SMA. M1 leg area sites and SMA sites were medial to the region shown enlarged (arrows). In PM, multiple sites were injected in the same session (see Table 1).

had to be removed from the rotation to permit the monkey to continue. Subsequently, intermittent failures of $2 \mathrm{f}, 1 \mathrm{e}, 3 \mathrm{e}$, and $4 \mathrm{e}$ occurred. Although the monkey continued to be able to perform more $2 \mathrm{f}$ trials successfully, movements $1 \mathrm{e}, 3 \mathrm{e}$, and $4 \mathrm{e}$ had to be removed from the rotation. Now with only $1 \mathrm{f}, 2 \mathrm{f}, 3 \mathrm{f}$, Wf, and We

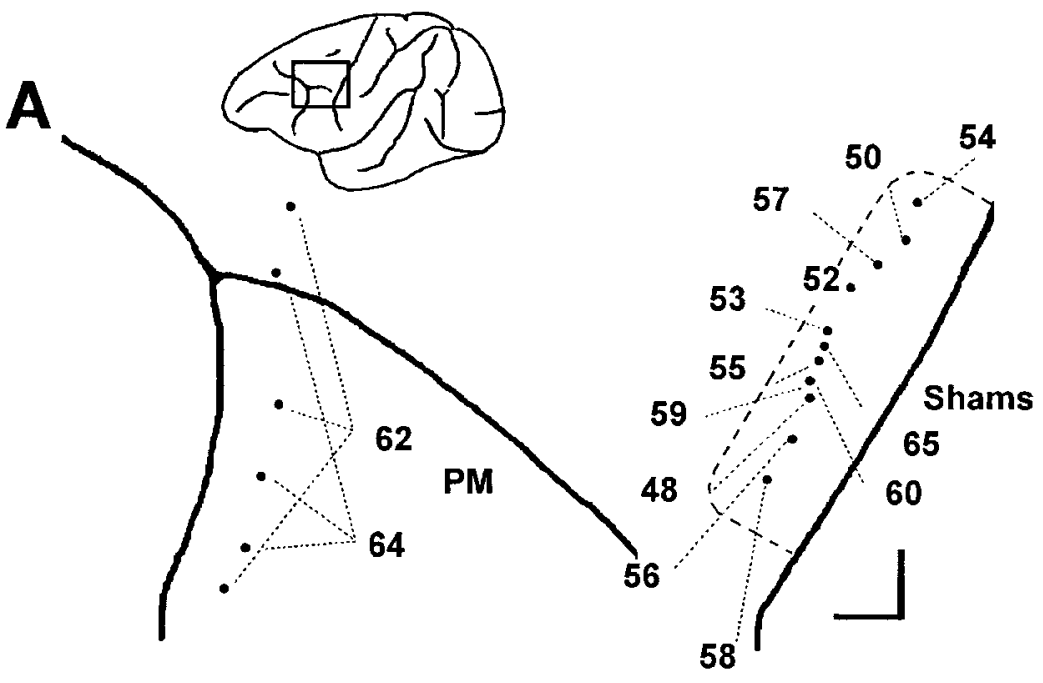

remaining in the rotation, consecutive correctly performed trials of each of these five movements occurred with fewer other instructed movements in between, reflected by an increased density of upward ticks for these five instructed movements after 1000 trials. Failures of $3 \mathrm{f}$ began to appear next. Finally, after a total of 


\section{Session 17}

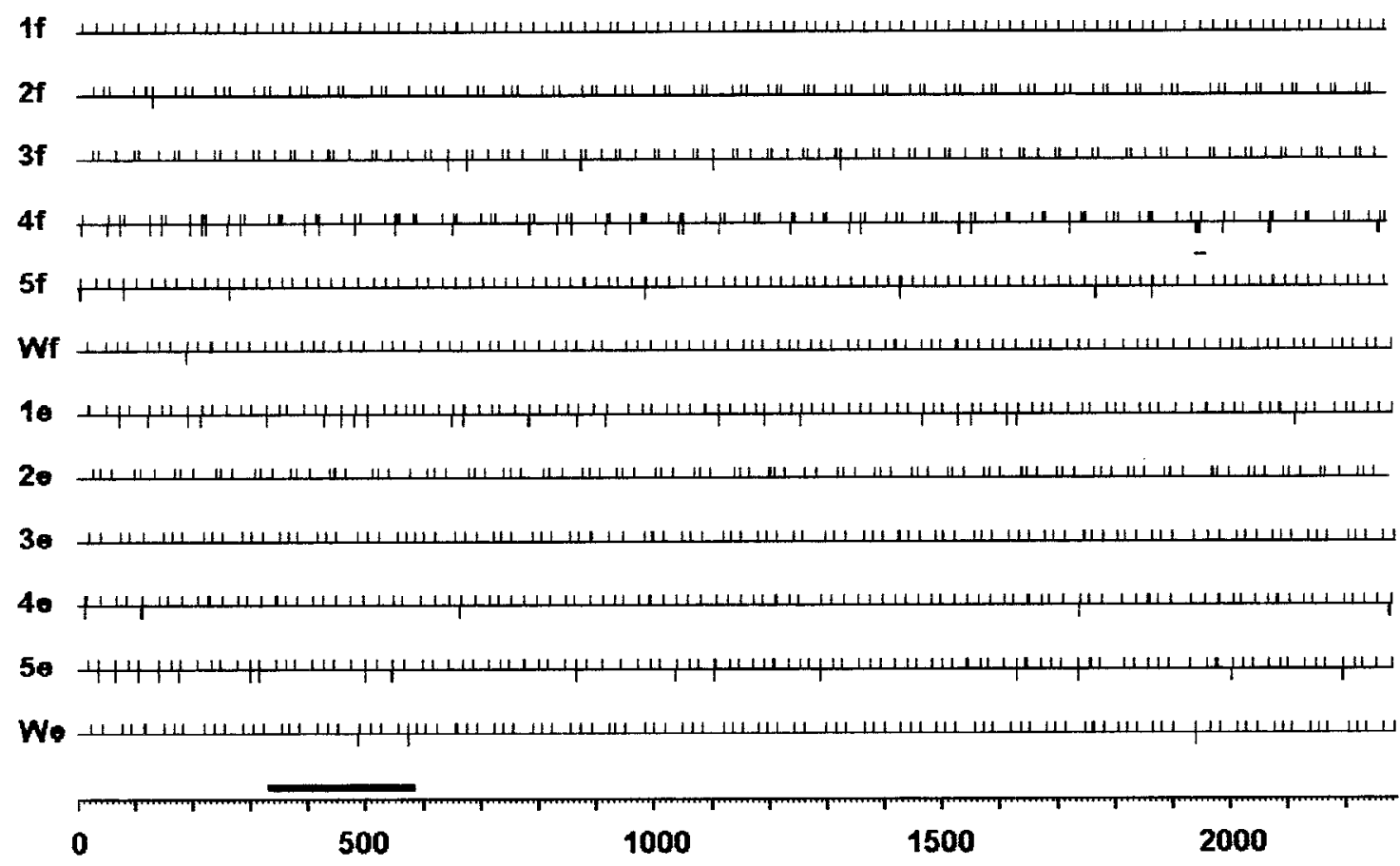

\section{Session 21}

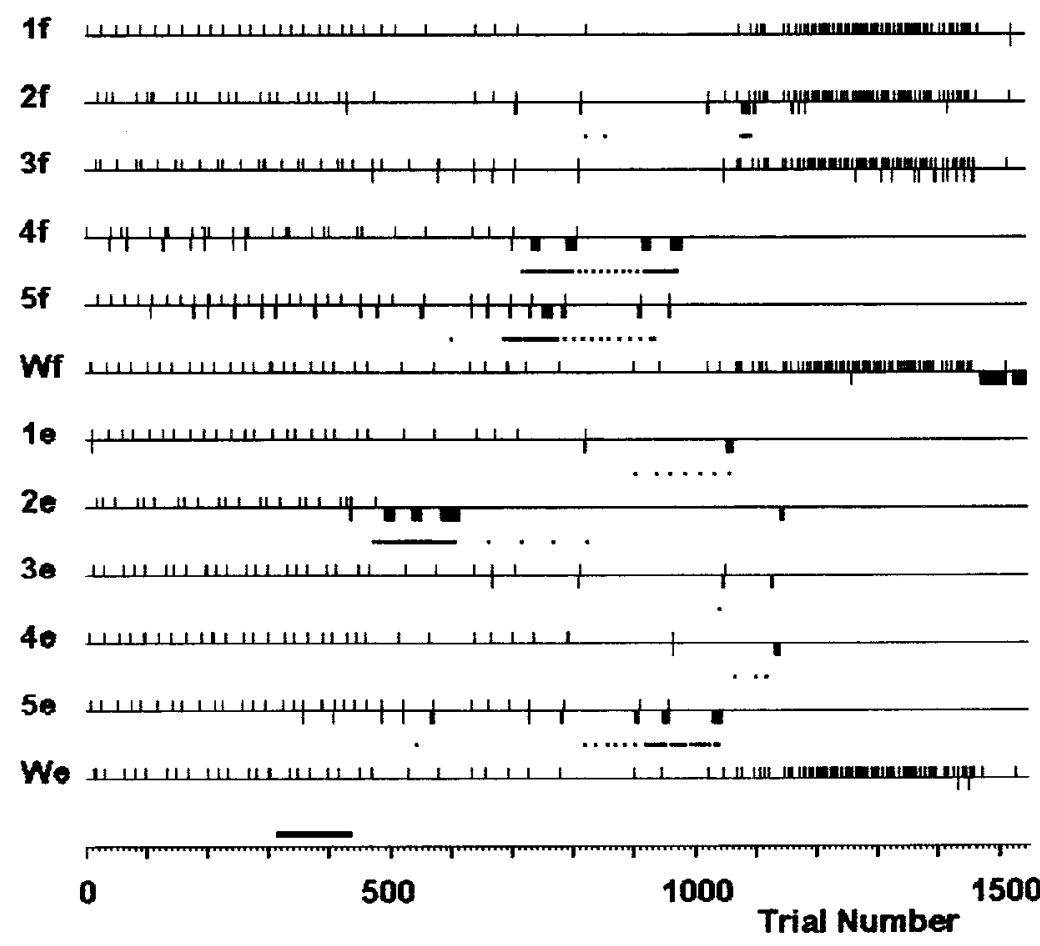

Figure 2. Success and failure of different instructed movements in two sessions. Horizontal lines for each of the 12 instructed movements are marked with upward ticks for each correctly performed trial and with downward ticks for each incorrectly performed trial of that instructed movement. Dots beneath these lines indicate the mean trial number of 10 consecutive trials of that instructed movement whenever 8 of the 10 were failures. Note that sequential ticks or dots occurring in the same row appear merged into a thickened tick or line segment, respectively, as minor divisions of the horizontal axis represent 10 sequential trials. A sham injection was performed in session 17, and a single muscimol injection was performed in session 21 , during the trials indicated by the bar just above the horizontal trial number axis for each session. The sham injection in session 17 did not alter the monkey's performance, whereas the muscimol injection in session 21 was followed by an increased failure rate for several instructed movements but not others. Session 17 spanned 93 min (injection, $13 \mathrm{~min}$ ); session 21 lasted $102 \mathrm{~min}$ (injection, $5 \mathrm{~min}$ ). 
102 min during which he performed $<1600$ trials, the monkey suddenly stopped performing Wf, presumably because of satiation and/or fatigue. Compared with session 17, in session 21 the monkey performed fewer trials in more time, in part because he waited longer on average between trials and in part because time was required to change the instruction sequence each time an instructed movement had to be removed from the rotation. Throughout session 21, however, few failures occurred on trials of instructed movements $1 \mathrm{f}$ or We.

As exemplified by sessions 17 and 21 from monkey $\mathrm{K}$, the visually cued, individuated finger movement task was sufficiently difficult that monkeys failed a number of trials at baseline, and sham injection of saline did not appear to affect this performance. Each monkey's baseline performance, and therefore success and failure rate, on particular instructed movements differed. Nevertheless, several minutes after injection of muscimol in the left M1 hand area, the monkey typically began to fail trials of some instructed movements. The frequency of failures on trials of certain instructed movements gradually increased, and eventually the monkey became unable to perform some of those movements but remained able to perform others.

To examine this impairment more concisely, we used failure of 8 of 10 consecutive trials of a given instructed movement as an empirical criterion of impaired performance of that particular movement. Other criteria, such as failure of 10 consecutive trials, generally were met once the monkey, having recognized his impairment, simply allowed trials of that particular instructed movement to time out rather than attempting to perform them. Still other criteria, based on deviation from the baseline mean error rate for each instructed movement, failed to reflect the fact that some instructed movements were genuinely more difficult than others for the monkey to perform at baseline. Although somewhat arbitrary, the criterion of 8 failures in 10 attempts correlated well with our impression that the monkey, although still motivated to attempt the particular instructed movement, was on the verge of becoming motorically unable to do so successfully.

Points at which this 8 of 10 consecutive failure criterion was met for each instructed movement are indicated in Figure 2 by dots beneath the rows of tick marks. A dot is positioned at the mean trial number of every 10 consecutive trials of a given instructed movement that included 8 failures. Note that because the successfully performed trials of a given instructed movement were followed by trials of other instructed movements, the 10 consecutive trials of one instructed movement initially were not 10 serial trials. The first dot indicating the mean trial number of the 10 trials therefore usually preceded the final series of sequential failures, consistent with our choice of a criterion representing the monkey's verging on being unable to perform the movement. The 8 of 10 consecutive failure criterion was met for only 1 instructed movement in session 17 (4f) but for 8 of the 12 instructed movements in session 21 (2f, 4f, 5f, 1e, 2e, 3e, 4e, and $5 \mathrm{e})$. For brevity, we will refer to instructed movements that met the 8 of 10 consecutive failure criterion as "failed" movements.

[In one situation, however, we did not consider the 8 of 10 criterion to indicate a failed movement. The monkey sometimes ended a session by suddenly not responding on multiple consecutive trials of a particular instructed movement, as happened for movement Wf in session 21 (Fig. 2). Because the monkey may have stopped working for motivational reasons, such as frustration, fatigue, or satiation, we do not consider an instructed movement that met the 8 of 10 criterion at the very end of a session to have been failed motorically.]
The failed instructed movements from each session are presented as a separate bar graph in Figure 3. The graph for each session has a bar for every failed movement. The height of each bar indicates the temporal sequence in which different instructed movements failed, from first (tallest bar) to last (shortest bar). In all three monkeys, more instructed movements failed after muscimol injections in the M1 hand area than after control injections. In monkey K, no more than one movement failed in control sessions (either saline injections in the M1 hand area or muscimol injections in other areas), whereas four to eight instructed movements failed after each muscimol injection. A notable exception was session 20, in which a low dose of muscimol $(1 \mu \mathrm{g})$ was injected, and only one movement subsequently failed. In monkey A, trained to perform only six movements, one to four instructed movements failed in different control sessions, whereas three to six instructed movements failed after muscimol injections in the M1 hand area. Indeed, within a central core of monkey A's M1 hand area-sessions 56, 48, 59, 55, and 53-five or six instructed movements failed after each injection, although only three or four did so after the four most medial and one most lateral injections. In monkey $\mathrm{H}$, four to seven instructed movements failed after each M1 hand area muscimol injection, whereas in the one control session (SMA), only two instructed movements were so affected.

Muscimol injection in the M1 hand area thus caused the monkey to fail some, but usually not all, instructed movements. In a given monkey, different instructed movements failed after different injections. Each of the five fingers was affected by some injection in each monkey (except for monkey A, in which digit 5 was not tested), indicating that muscimol injection in the M1 hand area could affect instructed movements of each digit. Little if any relationship was evident, however, between the location of the injection along the central sulcus and which instructed movement(s) failed. This is examined further in Table 2, where each failed movement in each session is indicated by *. Here, the M1 hand area sessions in each monkey have been arranged from top to bottom in order of the injection location from lateral to medial along the central sulcus. Somatotopic representation of the different fingers therefore should be apparent as a diagonal band of effects running from top left (thumb movements affected by lateral injections) to bottom right (little finger and wrist movements affected by medial injections). In monkey $\mathrm{K}$, although thumb flexion (1f) failed only after two lateral injections, thumb extension (1e) failed after all muscimol injections in the M1 hand area, including medial injections. Both little finger movements, $5 \mathrm{f}$ and $5 \mathrm{e}$, failed after the three more medial injections, but one or the other also failed after the three more lateral injections. No trends were evident for the other digits. In monkey $\mathrm{H}$, although firm conclusions could not be drawn with only three injection sites, no overt somatotopic segregation of failed movements was evident. No trend whatsoever was seen in monkey A, although digit 5 and the wrist were not tested.

The overall pattern of instructed movement failures after partial inactivation of the M1 hand area thus suggested distributed, rather than somatotopic, control of each individuated finger movement. This suggestion was supported further by instances in which particular instructed movements did not fail. Both in session 19 at the lateral end of monkey K's hand area and in session 16 at the medial end, for example, movements of digits 1, 2, and 5 failed, but movements of digits 3 and 4 did not fail in either of these sessions. If the fingers were controlled via an orderly somatotopic array in the M1 hand area, and if muscimol injection caused failure of movements of digits 1,2 , and 5, then movements 

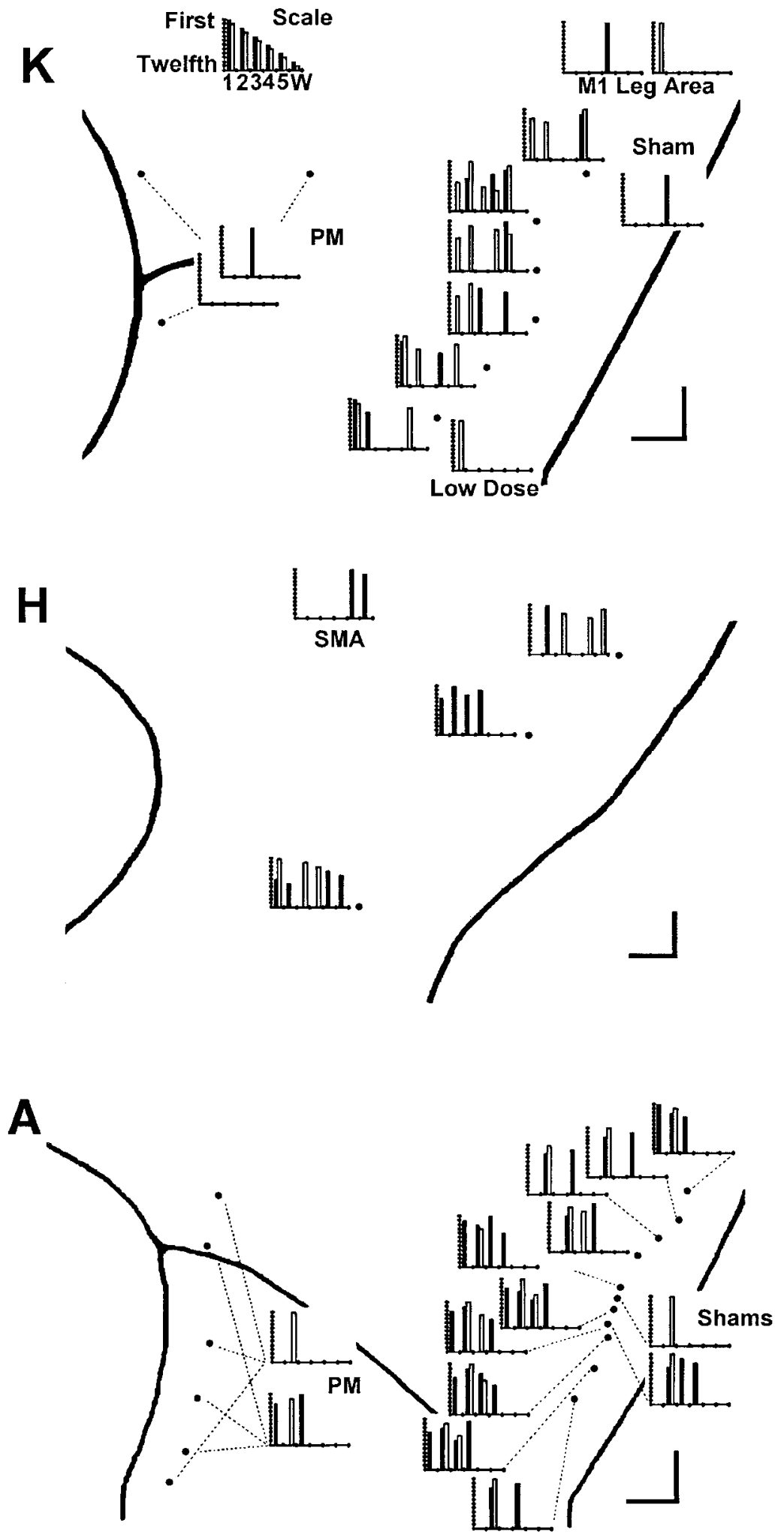

Figure 3. Failure on 8 of 10 consecutive trials. A bar graph for each session is used to show which instructed movements met the 8 of 10 consecutive failure criterion. Each bar graph has been positioned on the enlarged map of each monkey's left hemispheric surface close to the point at which the injection was made or else connected to it with a fine dashed line. (Three dashed lines indicate the three different locations injected during each PM session.) Each instructed movement that failed (met the 8 of 10 criterion) is shown as a bar positioned along the abscissa to indicate the instructed digit and shaded to indicate the instructed direction: filled, flexion; open, extension. The height of each bar indicates the serial order in which different instructed movements failed within each session from 1st (tallest) to 12th (shortest). The scale at top thus would illustrate an idealized result in which an injection placed laterally in the hand area impaired instructed movements starting with those of the thumb and spreading somatotopically to those of the little finger and wrist, with instructed flexion of each digit failing before instructed extension. Such a result was not obtained, however. Although in each monkey $(\mathrm{K}, \mathrm{A}$, and $\mathrm{H})$, muscimol injections in the $\mathrm{M} 1$ hand area caused more instructed movement failures than controls, which finger movements failed showed no systematic relationship to the location of the injection along the central sulcus. of digits 3 and 4 should have failed as well. Additional examples in which neither the flexion nor the extension movement of a particular digit failed despite failure of other digits flanking that digit on both sides can be found in several other sessions as well. Such gaps in the somatotopic order of affected digits are inconsistent with control of the fingers via a somatotopic array in M1, again indicating that control of each individuated finger movement is distributed in the hand area.

Furthermore, the flexion and extension instructed movements of a particular digit did not always fail together. In session 21 , for example, instructed movements $2 \mathrm{f}$ and $2 \mathrm{e}$ failed, $4 \mathrm{f}$ and $4 \mathrm{e}$ failed, and $3 \mathrm{e}$ failed, but $3 \mathrm{f}$ did not. If digits $2-4$ were controlled via a 
Table 2. Summary of effects

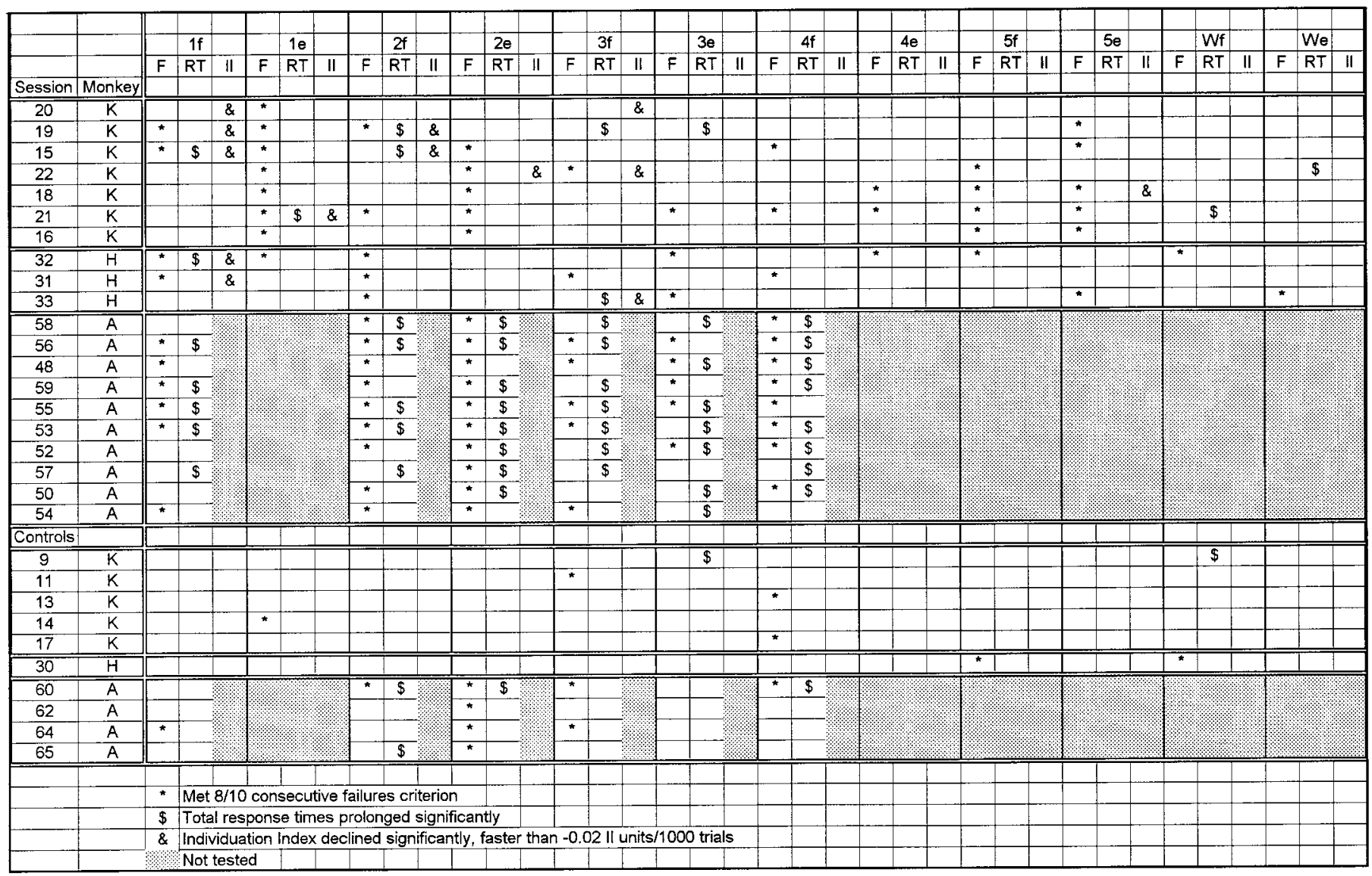

somatotopic array, and if both the flexion and extension movements of digit 2 and both movements of digit 4 failed, then both movements of digit 3 should have failed as well. Instances in which either the flexion or extension movement (but not both movements) of a particular digit failed can be found in most sessions from each of the three monkeys (although less commonly in monkey $\mathrm{A}$, in which for digits 1 and 4 only flexion movements were examined). These observations suggest that the contributions of M1 to the flexion versus the extension movements of a given digit were to some extent dissociable. Such dissociated impairment of flexion versus extension movement of a given digit, especially when that digit is flanked on both sides by digits with failed movements, again is inconsistent with somatotopic control of the fingers from M1. These observations provide further indications that the contribution of M1 to control of each individuated finger movement is distributed in the hand area.

Because muscimol diffuses to some extent through the cortex over time (Martin, 1991), focusing on the first movement to fail in each session (Fig. 3, tallest bar in each graph) might reveal a somatotopic trend. Indeed in monkey $\mathrm{K}$, 1f or 1e failed first after each of the three most lateral injections, 5e failed first after the most medial injection, and $2 \mathrm{e}$ failed first after the three injections located in the middle of the M1 hand area. An instructed movement of digit 3 or 4 never failed first, however, although the injections were spaced relatively evenly over the mediolateral extent of the hand area. In monkey $\mathrm{H}, 1 \mathrm{e}$ failed first after the most lateral injection, and $2 \mathrm{f}$ failed first after both of the two more medial injections. Although only three injections were made in monkey $\mathrm{H}$, instructed movements of digits 3-5 failed first in none of them, even the two relatively medial injections. In monkey A, movement $2 \mathrm{e}$ or, in one case, $3 \mathrm{f}$ failed first after all injections, except the most medial injection, in which $1 \mathrm{f}$ failed first, and the next to most lateral injection, in which 4 f failed first. Focusing on the first instructed movement to fail thus suggested a somatotopic gradient in monkey $\mathrm{K}$, with the thumb represented more intensely laterally and the little finger represented more intensely medially. Although a similar gradient might have existed in monkey $\mathrm{H}$, none was evident in monkey A. Moreover, in none of these monkeys was a somatotopic progression involving each of the five digits evident.

\section{Prolongation of response time}

Permanent lesions or reversible inactivations of the entire M1 hand area (Travis, 1955; Hamuy, 1956; Kubota, 1996; Rouiller et al., 1997), as well as lesions of the pyramidal tract (Beck and Chambers, 1970; Hepp-Reymond and Wiesendanger, 1972; Hepp-Reymond et al., 1974; Laursen, 1977), slow the performance of motor tasks. Fewer movements can be performed in a given period, and response times in discrete trials may be prolonged. Studies in which such slowing has been demonstrated previously have examined performance of natural tasks, such as retrieving food morsels or exerting force with precision pinch. Slowing of movements could cause failures in the present task, because the monkey was required to close the instructed switch within $700 \mathrm{msec}$ after the cue onset.

To determine whether such slowing occurred after the present muscimol injections and whether slowing affected all instructed 
movements or selectively affected some movements but not others, we measured the total response time for each correctly performed trial and sorted the response times according to the instructed movement. Total response time was defined as the time from the onset of the red LED instructional cue until closure of the instructed switch and thus included both the premovement reaction time and the movement time itself. We excluded from this analysis any trials immediately preceded by a failed trial, because in this circumstance the response time could be shortened by the monkey's knowledge that the same movement would be cued again after the failed trial.

Figure 4 shows a plot of response times on the remaining correctly performed trials of each instructed movement versus trial number in session 50. Before the muscimol injection in this session, monkey A closed the 4f switch 250-450 msec after the cue appeared. After muscimol was injected, however, $4 \mathrm{f}$ response times gradually increased. As the monkey became unable to perform $4 \mathrm{f}$ successfully, response times approached the $700 \mathrm{msec}$ limit imposed by the behavioral task. Although this trend was not necessarily linear, we calculated the slope of the best-fit line of response time versus trial number, starting from the beginning of the muscimol injection, as an empirical measure of the rate at which response times increased. For trials of $4 \mathrm{f}$, the response time increased at an average rate of $0.40 \mathrm{msec} /$ trial after the muscimol injection began until the monkey failed $4 \mathrm{e}$ (a slope significantly different from zero at $p<0.05$ after Bonferroni correction for six tests). Similarly in trials of $2 \mathrm{e}$, response times increased at 0.32 $\mathrm{msec} /$ trial after the muscimol injection began until the monkey failed $2 \mathrm{e}$. In trials of $3 \mathrm{e}$, response times also increased significantly but more slowly ( $0.09 \mathrm{msec} /$ trial $)$, and the monkey remained able to perform $3 \mathrm{e}$ throughout the session. Response times did not change significantly for movements $1 \mathrm{f}, 2 \mathrm{f}$, and $3 \mathrm{f}$, however, although the monkey failed $2 \mathrm{f}$ near the end of the session.

Figure 5 graphically displays such changes in total response times after each injection in monkey A, using a bar graph for each session to show the magnitude of significant slopes. The graph for each session has a bar for every instructed movement that showed a response time trend with slope significantly different from zero. The height of each bar indicates the regression slope of the trend in milliseconds per trial. Each muscimol injection in monkey A's M1 hand area was followed by significant prolongation of response times for some instructed movements but not others, except for session 53, in which response times increased slightly but significantly for all six instructed movements. In contrast, neither the sham injections in M1 nor the larger and more extensive muscimol injections in PM significantly prolonged any response times in monkey $\mathrm{A}$. In fact, response times of some movements shortened over the session, resulting in a significant negative slope.

Table 2 summarizes the instructed movements for which response times were significantly prolonged in each session in all three monkeys. Instructed movements in which the response times became prolonged after muscimol injections are indicated by $\$$. Prolonged response times were especially frequent in monkey A, occurring after every M1 hand area muscimol injection. Nevertheless, in all three monkeys, more instructed movements showed prolonged response times after muscimol injections in the M1 hand area than after control injections. In monkey $\mathrm{K}$, four of the seven M1 hand area muscimol injections prolonged the response times of $1-3$ of the 12 instructed movements performed. In contrast, of the five control injections in monkey $\mathrm{K}$ - the sham injection in the M1 hand area, two M1 leg area injections, and two
PM injections-only one PM session was followed by any significant prolongations of response time. In monkey $\mathrm{H}$, two of the three M1 hand area injections were followed by significant prolongations of response times in one movement, whereas the SMA injection produced no such change. The present muscimol injections in the M1 hand area thus prolonged the response times of some instructed movements but not others.

In monkey A, Figure 5 reveals little if any relationship between the location of the injection along the central sulcus and which response times of the digit were prolonged. Even focusing on the instructed movements with the most rapid prolongations of response times (tallest bars) did not reveal somatotopic trends. In Table 2, where the sessions in each monkey have been arranged such that a somatotopically ordered representation of the different fingers should be apparent as a diagonal band of significant effects running from top left (thumb movements affected by lateral injections) to bottom right (little finger and wrist movements affected by medial injections), no such trend was evident in the response time prolongations for any of the three monkeys.

\section{Decrease of individuation}

Loss of dexterity in fine finger movements is a well known result of lesions affecting the M1 hand area or corticospinal tract. In part, this may result from slowing of movements when speed is critical or weakness when strength is critical. In addition to slowness and weakness, voluntary movement of an affected body part often is accompanied by unintended simultaneous movement of other body parts. For example, in retrieving food morsels from small wells, instead of the precision pinch they normally use, monkeys with M1 lesions (Passingham et al., 1983) or corticospinal lesions (Lawrence and Kuypers, 1968; Woolsey et al., 1972; Schwartzman, 1978; Chapman and Wiesendanger, 1982) make raking movements using all the fingers. In the present individuated finger movement task, this loss of relatively independent finger movements could appear as excessive movement of noninstructed digits when the monkey attempted to move the instructed digit. Excessive movement of noninstructed digits could close the wrong switch, either before closure of the instructed switch or during the final hold period, resulting in a failed trial.

To determine whether noninstructed digits moved excessively after muscimol injections, we computed previously described (Schieber, 1991) relative motion coefficients and Individuation Indexes on a trial-by-trial basis for each instructed movement in each session (see Materials and Methods). Data for this analysis were available for monkeys $\mathrm{K}$ and $\mathrm{H}$ but not for monkey A. Figure 6 shows an example from session 15. The top two panels show data from two different trials of movement 1f, one before the muscimol injection (Fig. 6A), one after (Fig. 6B). In each of these panels, the normalized position of each of the five fingers and the wrist has been plotted against the simultaneous normalized position of the instructed digit, digit 1 . Whereas before the muscimol injection, all the noninstructed digits remained relatively stationary as digit 1 flexed (Fig. $6 A$ ), after the muscimol injection digits 2 and 4 extended as digit 1 flexed (Fig. $6 B$ ). In these two panels, the plot of digit 1 against itself forms a line with slope $=1.0$. The plots of other digits against digit 1 also have linear components, indicating that the motion of the noninstructed digits followed time courses similar to that of the instructed digit. We therefore used the slope of the best-fit line for each plot of noninstructed versus instructed digit position as a coefficient of the motion of each noninstructed digit relative to that of the instructed digit. This coefficient (slope) was close to 0 if the noninstructed digit did 
Figure 4. Prolongation of response times. The response times, from cue onset to switch closure, on successful trials have been sorted according to instructed movement (1f, 2f, 3f, 4f, 2e, and $3 e)$ and plotted as a function of sequential trial number in session 50. Muscimol was injected as monkey A performed trials 317-462 (black bar beneath each plot). Subsequently, response times became progressively longer in trials of $4 \mathrm{f}, 2 \mathrm{e}$, and $3 \mathrm{e}$, and linear regression showed slopes significantly different from zero, whereas response times in trials of $1 \mathrm{f}, 2 \mathrm{f}$, and $3 \mathrm{f}$ remained stable. Shortly before 1000 trials, monkey A became unable to permovements were removed from the rotation, making subsequent trials of $1 \mathrm{f}$, $2 \mathrm{f}, 3 \mathrm{f}$, and $3 \mathrm{e}$ relatively more frequent. The total elapsed time in session 50 was $72 \mathrm{~min}$, and the injection was performed over $5 \mathrm{~min}$. form $4 \mathrm{f}$ and $2 \mathrm{e}$, and these instructed
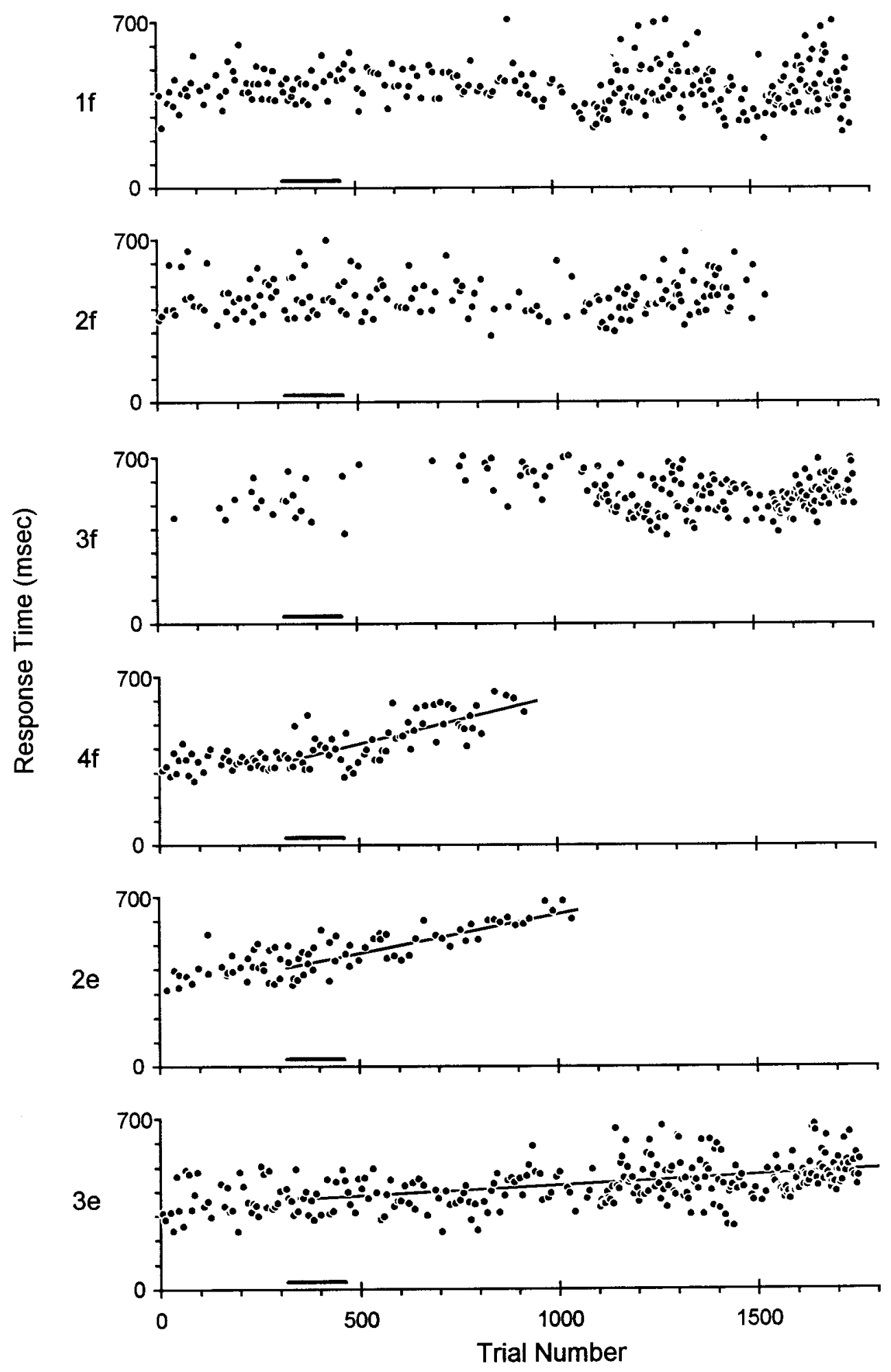

not move but was closer to 1 the more the noninstructed digit moved along with the instructed digit or closer to -1 the more the noninstructed digit moved in the opposite direction.

Figure $6 C$ plots these relative motion coefficients for each correctly performed trial of $1 \mathrm{f}$ in session 15 . The two trials shown in Figure 6, $A$ and $B$, are indicated with arrowheads. The coefficient for digit 1 , the instructed digit, by definition was unity for all 1f trials. Before the muscimol injection, the coefficients for the other, noninstructed digits all were close to 0 , indicating little if any motion of other digits as the thumb flexed. After the injection, however, the coefficients of noninstructed digits deviated progressively away from 0 , reflecting increasing amounts of movement of these noninstructed digits in the same (positive values) or opposite (negative values) direction as the flexing thumb.

An overall Individuation Index for each correctly performed instructed movement then was computed as $1-$ mean of the 


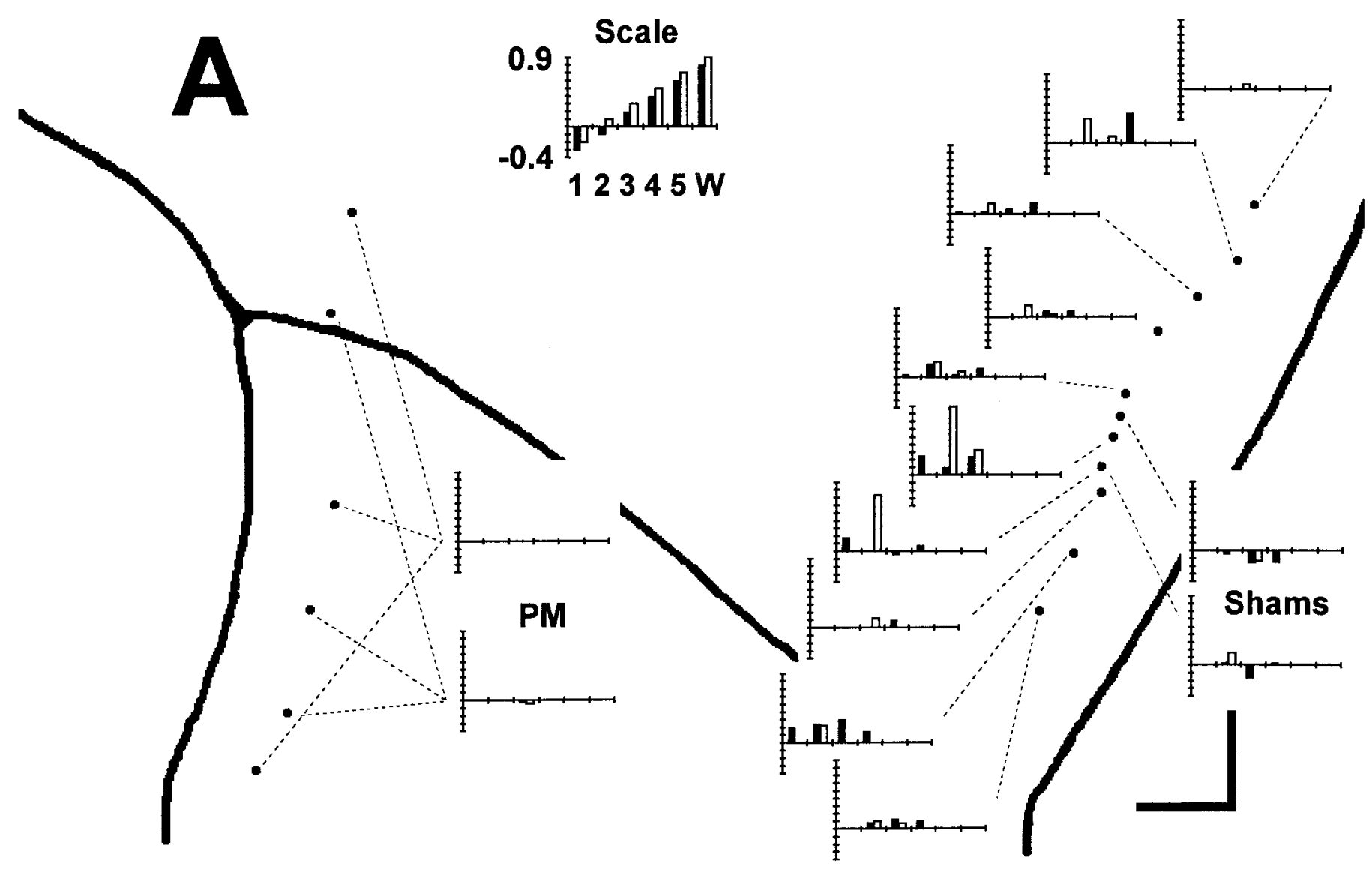

Figure 5. Response time changes in each session in Monkey A. A bar graph for each session in monkey A is used to show which instructed movements had progressive changes in response time with slope significantly different from zero. Bar graphs are positioned and formatted as in Figure 3 , but here the height of each bar indicates the slope of the best-fit line of response time versus trial number in units of milliseconds per trial. The scale at top thus would illustrate an idealized result in which an injection placed medially in the hand area prolonged response times most dramatically for wrist and little finger movements and less for ring and middle finger movements, whereas response times of thumb movements shortened. Such a result was not obtained, however. Rather, different muscimol injections prolonged the response times of some instructed movements but not others in any given session. Which movements were affected showed little relation to the location of the injection along the central sulcus.

absolute values of the coefficients of the noninstructed digits (see formula in Materials and Methods). This Index was closer to 0 the more the noninstructed digits moved and closer to 1 the less the noninstructed digits moved. Figure $6 D$ plots the Individuation Index for each correctly performed trial of $1 \mathrm{f}$ in session 15 , using the coefficients shown in Figure $6 \mathrm{C}$. Before the muscimol injection, the Individuation Index was consistently close to 1 . After the injection, the Individuation Index fell progressively, reaching 0.72 on the last correctly performed $1 \mathrm{f}$ trial. Figure $6 E$ shows the trial-by-trial Individuation Index of successful $4 \mathrm{e}$ trials from the same session. Before muscimol injection, the $4 \mathrm{e}$ Individuation Index was $\sim 0.75$. Although the Individuation Index for $1 \mathrm{f}$ trials fell after the muscimol injection, the Individuation Index for $4 \mathrm{e}$ remained stable.

To determine whether the Individuation Index decreased systematically after muscimol injections in the M1 hand area, we performed a separate linear regression of Individuation Index versus trial number for each instructed movement, from the first trial performed during the injection through the last correctly performed trial of each instructed movement. Although these trends again were not necessarily linear, we used the probability of the slope being significantly different from zero $(p<0.05$ after Bonferroni correction) as an indicator that the trend was significant, and we used the slope of the best-fit line as a measure of the rate at which the Individuation Index decreased. Because changes in individuation to some degree might occur as the monkey became satiated or fatigued over a long session, we performed the same analysis on the control sessions in each monkey.

Figure 7 displays as a bar graph the significant changes in Individuation Index from each muscimol injection session in monkey K. The graph for each session has a bar for every instructed movement that showed an Individuation Index trend with slope significantly different from zero. The height of each bar indicates the regression slope of the trend in Individuation Index units per 1000 trials. In monkey K, the fastest rate of Individuation Index decrease observed in control sessions was -0.02 units/ 1000 trials. In comparison, each M1 hand area muscimol injection except for one at the medial edge was followed by faster rates of Individuation Index decrease for some movements, ranging from -0.03 to -0.17 units/1000 trials. (Increases in the Individuation Index for other instructed movements also occurred, as failed movements were removed from the rotation, reducing the set of different movements the monkey was required to perform.) In monkey $\mathrm{H}$ (data not shown), no significant decreases of Individuation Index occurred in the single control session, but decreases ranging from -0.01 to -0.05 units/1000 trials occurred after each of the three M1 hand area injections. Muscimol injection in a given session thus caused progressive increases in the motion of 
Figure 6. Changes in individuation in a single session. $A$, $B$, Plots of normalized finger positions $(0$, position at extension switch closure; 100, position at flexion switch closure) from two successful trials of instructed movement $1 \mathrm{f}$, one performed before $(A)$ and the other after $(B)$ the muscimol injection in session 15. In these two panels, the normalized position of each digit and of the wrist has been plotted as a function of the simultaneous normalized position of the instructed digit, digit 1 . Whereas the noninstructed digits did not move as the monkey flexed his thumb before the muscimol injection $(A)$, digits 2 and 4 extended as the monkey flexed his thumb after the injection $(B)$. $C$, Relative motion coefficients (ordinate) derived from the slopes of such plots for each digit in each correctly performed trial of 1f in session 15. The single trials shown in $A$ and $B$ are indicated here by arrowheads. After the muscimol injection (bar at bottom), coefficients for noninstructed digits diverged from their near-zero baselines. $D$, Individuation Index calculated from these coefficients for each successful if trial. $E$, Individuation Index for trials of $4 \mathrm{e}$ in the same session. The Individuation Index of $1 \mathrm{f}$ trials decreased progressively after the muscimol injection, whereas the Individuation Index for $4 \mathrm{e}$ trials remained stable. The total elapsed time in session 15 was $100 \mathrm{~min}$ (injection, $8 \mathrm{~min}$ ).
A

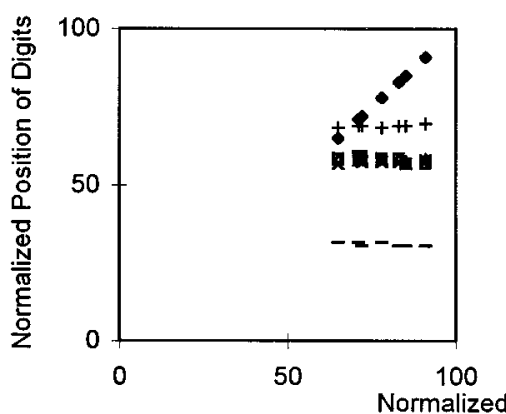

B

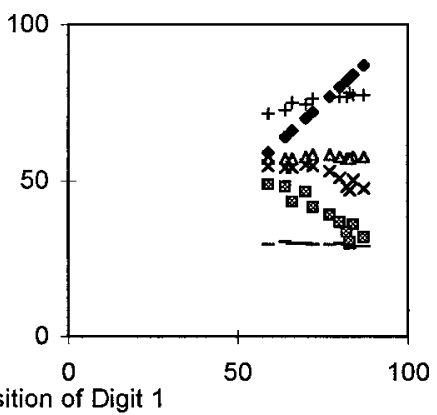

C

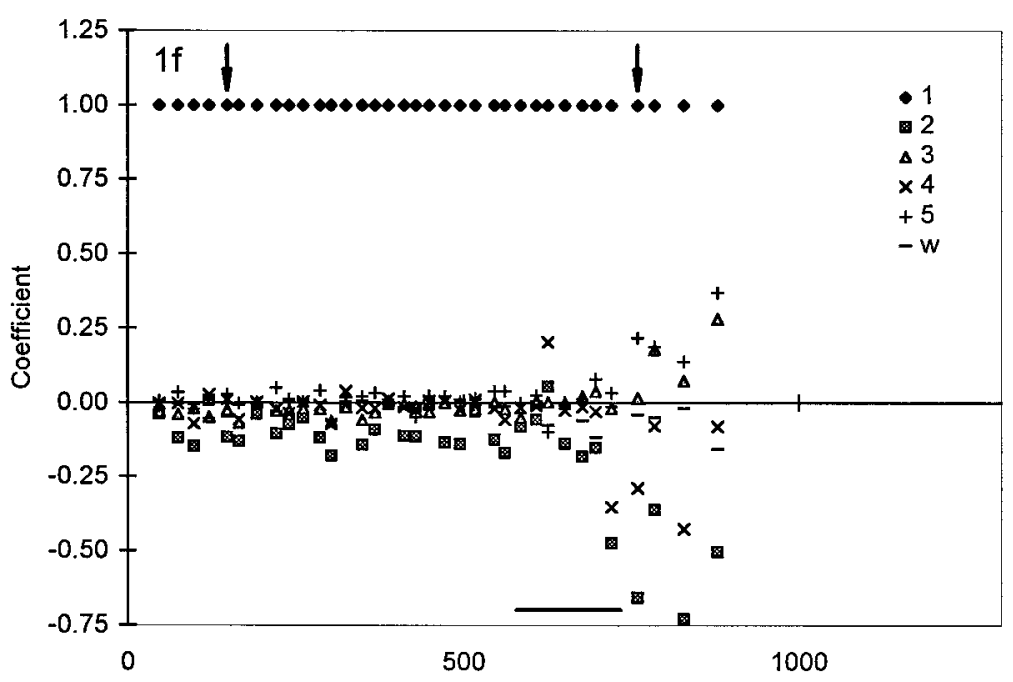

D

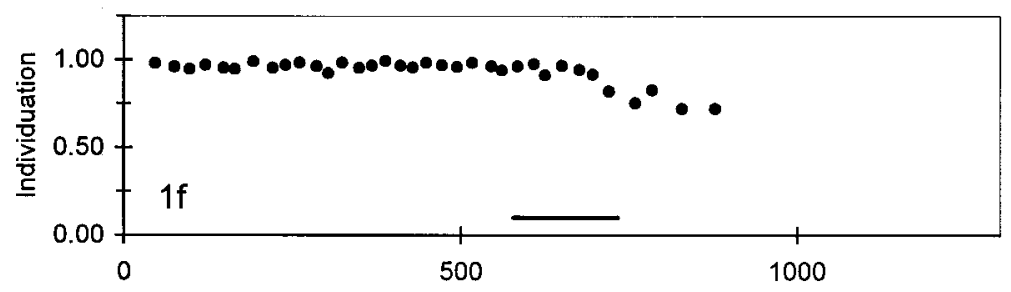

E

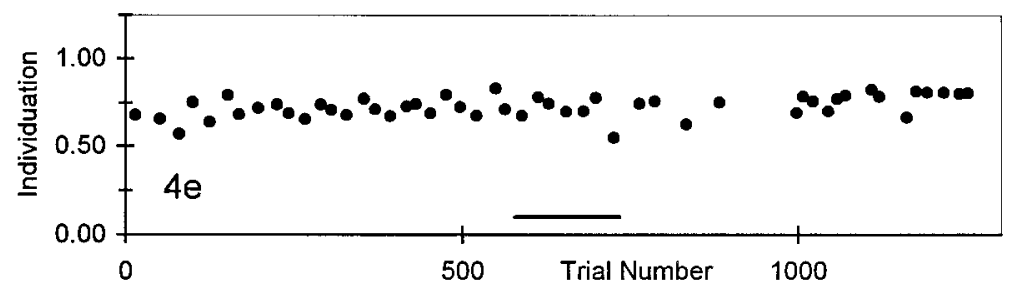

noninstructed digits during certain instructed movements but not during other instructed movements. Consequently, the Individuation Index for some instructed movements decreased progressively, whereas the Individuation Index for other instructed movements remained stable.

No relationship was evident, however, between the location of the muscimol injection along the central sulcus and the instructed movements that showed a decrease in individuation (Table 2, \&). In monkey $\mathrm{K}$, although the individuation of $1 \mathrm{f}$ was most profoundly affected after the three most lateral injections, 1e was even more profoundly affected after a relatively medial injection. In monkey $\mathrm{H}$, although $3 \mathrm{f}$ was affected by the most medial injection, 1f was affected by the other two injections, separated by 4-5 mm along the central sulcus.

\section{Combinations of effects}

Table 2 summarizes the observed effects in each session: which instructed movements failed, which showed significant prolongation of response time, and which showed significant decreases in Individuation Index beyond the -0.02 units/1000 trials observed in controls. A wide variety of the possible combinations of these effects was observed. For some instructed movements in some sessions (e.g., for 1f in session 15) all three effects were observed together; i.e., muscimol injection was followed by concurrent prolongation of response time and decrease in individuation on successful trials of 1f, until finally 8 of 10 consecutive $1 \mathrm{f}$ trials were failed. In other instances, however, instructed movements failed without ante- 

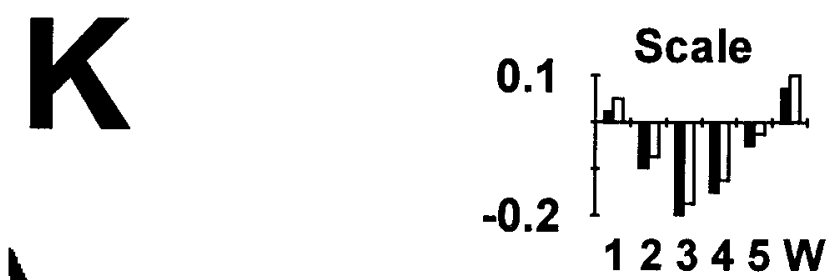

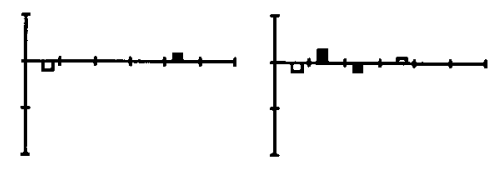

M1 Leg Area
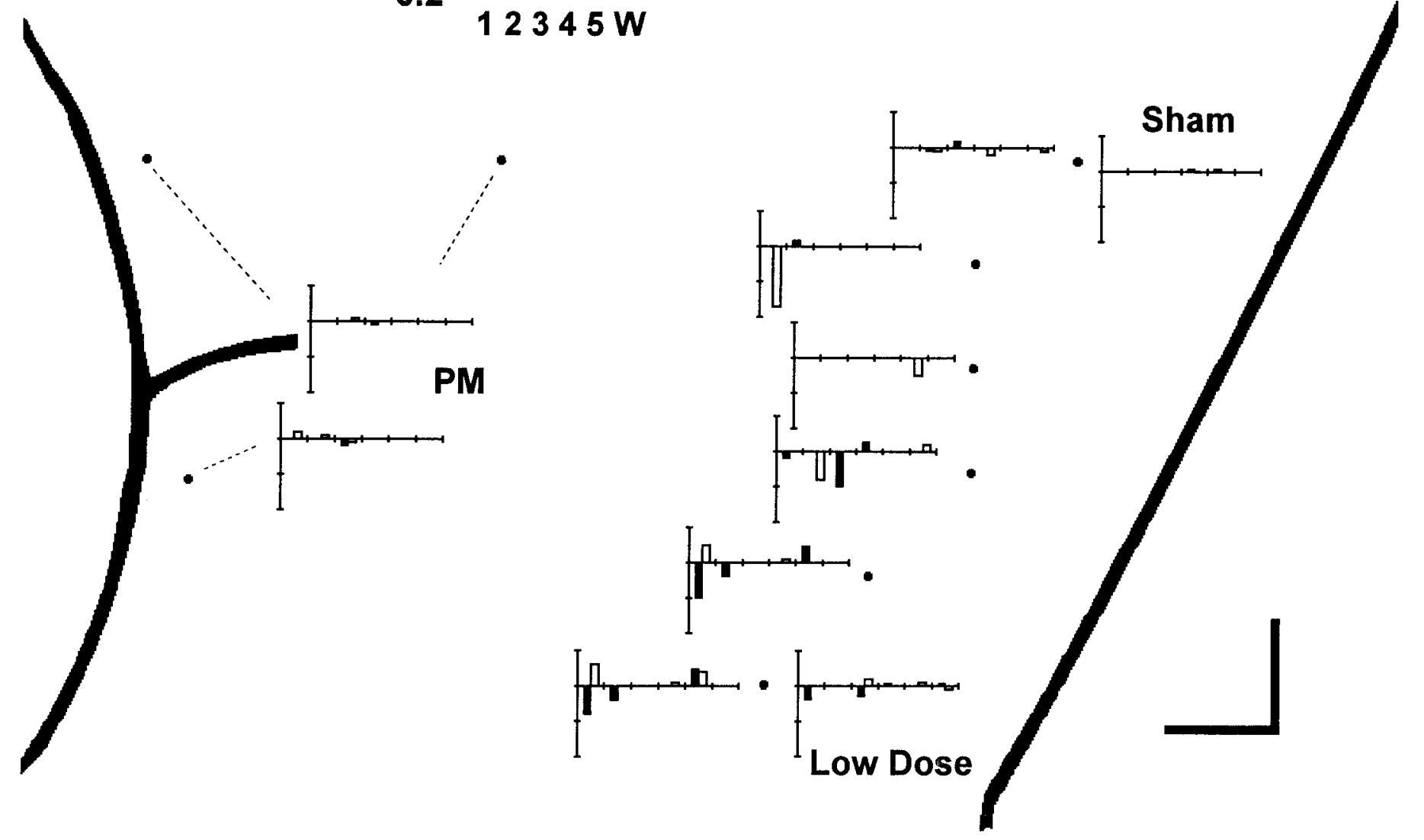

Figure 7. Individuation Index changes in each session in monkey K. A bar graph for each session in monkey K is used to show which instructed movements had progressive changes in Individuation Index with slope significantly different from zero. Bar graphs are positioned and formatted as in Figure 3, but here the height of each bar indicates the slope of the best-fit line of Individuation Index versus trial number, in Individuation Index units per 1000 trials. The scale at top thus would illustrate an idealized result in which an injection placed centrally in the hand area caused the greatest Individuation Index decrease for movements of the middle finger and less for index and ring finger movements, whereas Individuation Indexes of thumb and wrist movements increased. Such a result was not obtained, however. Rather, different muscimol injections impaired individuation of some instructed movements but not others in any given session. Which movements were affected showed little relation to the location of the injection along the central sulcus.

cedent prolongation of response time or decrease in individuation (e.g., $4 \mathrm{f}$ in session 15 ). In still other instances, response times became prolonged and/or individuation decreased, although the instructed movement never met the 8 of 10 consecutive failure criterion (e.g., $2 \mathrm{f}$ in session 15). Moreover, in some instances response times became prolonged without decreases in individuation (e.g., $3 f$ in session 19), or, vice versa, individuation decreased without prolongation of response time (e.g., $3 \mathrm{f}$ in session 20). That the different effects observed were not tightly linked suggests that prolongation of response time and decrease in individuation may reflect dissociable aspects of M1 dysfunction, and that factors other than those evaluated here may have contributed to instructed movement failure as well.

\section{Use of the fingers in retrieving food morsels}

Once the monkey had become either unable to perform any instructed movement or else satiated with water rewards, its hand was removed from the manipulandum and examined clinically.
After muscimol injections that produced impairment in the visually cued finger movement task, this examination typically revealed qualitatively decreased tone in the fingers, weakness of power grip, and some degree of finger and wrist drop, none of which was found after control injections. The monkey then was removed from the primate chair, and its ability to retrieve food morsels from the large and small food wells was examined.

In retrieving food morsels before muscimol injections, the monkey's hand typically preshaped, with extension of the index finger and flexion of the middle, ring, and little fingers, as the arm reached to either well. All fingers then entered the larger well, and a precision pinch was used to pick up the food morsel. Only the index entered the smaller well, and flexion of the index finger then was used to pull the food morsel to the lip of the well, where it was grasped in a precision pinch against the thumb. Similar patterns were observed in the right hand after the control injections and in the left hand after all injections.

In contrast, after muscimol injection in the left M1 hand area, 
the right hand failed to preshape. When retrieving food morsels from the larger well, all fingers still were able to enter in a partially flexed posture, and a raking motion was used to remove the food morsel, which might be held in the palm by flexing all the fingers. Often, however, the fingers failed to hold the food morsel, which fell to the ground after reaching the edge of the well. Occasionally the food morsel lodged between the lateral surfaces of two adjacent fingers and was held successfully. Retrieving food morsels from the small well was much more difficult after muscimol injection. Because the hand failed to preshape, the middle, ring, and little fingers remained extended and bumped into the Lucite surface next to the well, preventing the index finger from entering. The deficits produced by the present muscimol injections thus were not limited to the overtrained performance in the visually cued, individuated finger movement task. The present injections also impaired performance of a natural use of the fingers, producing deficits qualitatively similar to those described in previous studies involving more extensive permanent lesions, reversible inactivation of $\mathrm{M} 1$, or lesions of the corticospinal tract. Moreover, similar deficits in retrieving food morsels were observed after all muscimol injections in the M1 hand area, regardless of mediolateral location along the central sulcus.

\section{DISCUSSION}

\section{Partial inactivation of the M1 hand area}

We made single injections of muscimol that produced only partial inactivation of the M1 hand area. Although each injection impaired the monkey's ability to perform some individuated finger and wrist movements, others remained unimpaired. In different sessions, however, all the instructed movements were affected in one way or another. Furthermore, although each injection also impaired the monkey's performance in retrieving food morsels, this impairment appeared to be quantitatively less profound than that described after total structural ablation of the M1 hand area (Fulton and Kennard, 1932), lesions of the pyramidal tract (Lawrence and Kuypers, 1968), or more extensive inactivation with multifocal injections of lidocaine or larger doses of muscimol (Kubota, 1996; Rouiller et al., 1997). We infer that each muscimol injection affected the M1 hand area only partially.

Comparing the extent of the macaque M1 hand area with the extent of muscimol diffusion measured in rats suggests that the injected muscimol diffused over only part of the M1 hand area. The region of M1 containing neurons that discharge in relation to finger movements, and from which ICMS evoked finger movements, extended $\sim 6-9 \mathrm{~mm}$ along the central sulcus in each of our monkeys. In comparison, $1 \mu \mathrm{g}$ of muscimol in $1 \mu \mathrm{l}$ injected in rat cortex has been shown to spread in 120 min (longer than our experimental sessions) over an average radius of $<2 \mathrm{~mm}$ and to decrease glucose utilization over only a $3 \mathrm{~mm}$ radius (Martin, 1991). If such a muscimol injection at the lateral edge of the monkey hand area affected cortex within a $3 \mathrm{~mm}$ radius, somewhat less than half the hand area would have been affected. Although we used a higher concentration of muscimol $(5 \mu \mathrm{g}$ in 1 $\mu \mathrm{l})$, this is unlikely to have resulted in a substantially more widespread effect. In the three sessions in which we injected even more muscimol in the hand area (sessions 31, 32, and 59), the effects were not appreciably greater, suggesting that $5 \mu \mathrm{g}$ produced a relatively complete local effect. Most likely, then, each muscimol injection spread through only part of the M1 hand area.

\section{Motor abnormalities resulting from M1 lesions}

Motor abnormalities resulting from lesions of M1 or of the corticospinal tract are well known to neurological clinicians. The ability to use fine finger movements in performing tasks such as buttoning buttons or tying shoelaces is first lost and last recovered after small lesions. Performance of such tasks lacks both strength and dexterity. Voluntary attempts to move a single finger often result in excessive motion of several digits, the wrist, and even more proximal parts of the arm. Larger lesions produce overt weakness of the hand and fingers.

Similar experimental lesions in subhuman primates impair the use of relatively independent finger movements in retrieving food morsels from small wells (Lawrence and Kuypers, 1968; Nudo and Milliken, 1996). The forces that can be exerted in precision grip are reduced, and the rate of force change is slowed (HeppReymond and Wiesendanger, 1972). Moreover, response times are prolonged, both in the premovement period from cue to movement onset and in the movement period itself (Travis, 1955; Hepp-Reymond and Wiesendanger, 1972; Hoffman and Strick, 1995; Kubota, 1996). Consequently, retrieval of food morsels is less efficient (Hamuy, 1956; Nudo and Milliken, 1996; Nudo et al., 1996; Rouiller et al., 1997).

The weakness and slowness that result from ablation or inactivation of M1 correlate well with physiological properties of M1 neurons. The discharge frequencies of M1 neurons in a variety of experimental paradigms correlate with the force exerted in a voluntary movement and the rate of change of force, as well as with other kinematic parameters such as joint position and velocity (Evarts, 1968; Smith et al., 1975; Hepp-Reymond et al., 1978; Georgopoulos et al., 1992; Ashe, 1997). When M1 ablation or inactivation eliminates the bursts of corticospinal discharge that participate in the rapid recruitment of spinal motoneurons during a prompt movement, the resulting muscle contractions will be slow to develop and will reach peak forces lower than normal. Because this reduced force will act against unchanged inertial and viscous loads, the affected body part will move more slowly, and its movement may fall short of the intended excursion. Voluntary movement thus will be weak when tested isometrically and slow when tested isotonically. These deficits could have resulted in the prolongation of response times observed in successful trials of the present individuated finger movement task and eventually in performance failure. Although weakness and slowness might be dissociable deficits, our paradigm would not distinguish them.

Weakness and/or slowness, however, cannot explain the progressive decreases observed in individuation. Decreases in the Individuation Index reflect increased active movement of noninstructed digits occurring while the monkey attempted to move the instructed digit. Increased movement of nearby fingers also contributed to the deficit observed in the natural performance of retrieving food morsels from the small food well. Normally the monkey's index finger extended alone as the hand preshaped in approaching the food wells. After muscimol injection, however, the middle, ring, and little fingers extended along with the index finger as the hand preshaped, precluding smooth entry of the index finger into the smaller well. This increased active movement of nearby body parts, which contributes significantly to the loss of dexterity that follows M1 ablation, suggests that one aspect of the normal function of M1 may be to individuate movements by actively minimizing the motion of noninstructed digits. M1 normally might minimize unwanted motion in part through inhibition mediated by horizontal intracortical connections (Huntley and Jones, 1991; 
Jacobs and Donoghue, 1991; Keller and Asanuma, 1993; Ziemann et al., 1996), and in part through simultaneous activation of multiple muscles whose net effect moves one digit while minimizing motion of the others (Schieber, 1990, 1995).

The present findings also suggest that decrease in individuation is dissociable from weakness and slowness. The deficits in a given instructed movement produced by M1 inactivation often occurred together. After muscimol injection in session 15, for example, thumb flexions (1f) showed (1) decreased individuation, (2) prolonged response times, and eventually (3) failure in 8 of 10 consecutive trials. But at least as often a given instructed movement showed only one or two of these three abnormalities. Failing 8 of 10 consecutive trials without antecedent changes in response time or individuation in theory could have resulted if the latter two abnormalities developed so rapidly that too few successful trials occurred for trends to attain significance before complete failure set in. Conversely, mild prolongation of response time and mild decrease in individuation could have occurred in parallel, although never becoming severe enough to cause failure. If enough successful trials were available to demonstrate a significant prolongation of response time, however, enough successful trials should have been available to demonstrate a decrease in individuation as well. The finding that response time for a particular instructed movement could be prolonged without a simultaneous decrease in individuation or, vice versa, that individuation could decrease without concurrent prolongation of response time, suggests that these two abnormalities were to some extent dissociable. If we view response time prolongation as the result of weakness and/or slowness of the motion of the instructed digit and view decreased individuation as the result of inadequate stabilization of noninstructed digits, then our observations support earlier suggestions that M1 may have dissociable systems for active control of intended movements versus active stabilization (Humphrey and Reed, 1983).

\section{How somatotopic is the M1 hand area?}

If the present muscimol injections affected only part of the M1 hand area, and if different fingers were controlled via a mediolateral somatotopic array of finger representation in M1, then which finger movements were impaired should have been systematically related to the mediolateral location of the muscimol injections. But only when focusing on the first instructed movement to fail 8 of 10 consecutive trials did we note a trend in one monkey $(\mathrm{K})$ for thumb movements to fail first after lateral injections and little finger movements to fail first after medial injections. Although index finger movements were affected first by injections in between, no injections first affected movements of the middle or ring finger. Moreover, two observations were made in many sessions that appear inconsistent with a well ordered somatotopic representation of the different fingers: (1) the movements of a given finger could be unimpaired even though the movements of neighboring fingers were impaired; and (2) the flexion movement or extension movement of a finger could be impaired selectively. In general, which finger movements were affected by which muscimol injections appeared stochastic, with little if any systematic relation to the mediolateral location of the muscimol injection along the central sulcus.

Our findings are consistent with a growing body of experimental evidence indicating that the M1 hand area is not so somatotopically organized as suggested by the homunculus and simiusculus. This evidence can be summarized in four major points (for more detailed review, see Hepp-Reymond, 1988; Porter and
Lemon, 1993). First, output converges from a large territory in the M1 hand area onto any given hand muscle, and the territories that provide output to any two hand muscles overlap extensively (Andersen et al., 1975; Humphrey, 1986; Sato and Tanji, 1989; Donoghue et al., 1992). Similarly, the territory from which stimulation evokes movement of any given finger is large and overlaps extensively with the territory from which stimulation evokes movement of any other finger (Penfield and Boldrey, 1937; Woolsey et al., 1951, 1979; Kwan et al., 1978; Gould, et al., 1986). Second, single M1 neurons have outputs that diverge to the motoneuron pools of multiple muscles, which has been shown both anatomically (Shinoda et al., 1981) and physiologically (Cheney and Fetz, 1985; Buys et al., 1986). Third, horizontal interconnections linking the entire M1 hand area are provided by axons of local neurons and axon collaterals of corticofugally projecting neurons (DeFelipe et al., 1986; Huntley and Jones, 1991; Keller and Asanuma 1993). And fourth, although some studies in humans suggest broadly overlapping gradients of activation (Grafton et al., 1993; Kleinschmidt et al., 1997), studies in both monkeys and humans show that activation is distributed throughout the M1 hand area whenever any finger movement is made (Schieber and Hibbard, 1993; Sanes et al., 1995). The present findings make the additional point that which finger movements are impaired by partial inactivation of the M1 hand area is unrelated to the mediolateral location of the inactivation.

Why should the M1 hand area have a distributed organization for control of the different fingers, rather than a somatotopic representation? We suggest three potential reasons. First, in the somatotopic representation of the primary somatosensory cortex (S1) and in the retinotopic organization of the primary visual cortex (V1), two-dimensional receptor sheets are mapped onto the two-dimensional cortical surface. But movements and muscles exist in three dimensions and cannot be mapped isomorphically onto a two-dimensional cortex. Second, a well ordered representation such as that of S1 or V1 may facilitate interactions between nearby elements but at the same time would provide less facilitation of interactions between nonadjacent elements. A distributed organization would facilitate more evenly all possible interactions between different fingers. And third, movement of a finger does not occur in isolation (Schieber, 1991; Soechting and Flanders, 1997) but, rather, requires simultaneous control of the entire hand, involving contraction of many muscles acting on different fingers (Beevor, 1903a,b; Schieber, 1995). Such control may be achieved most efficiently by the distributed organization of the M1 hand area.

\section{REFERENCES}

Andersen P, Hagan PJ, Phillips CG, Powell TP (1975) Mapping by microstimulation of overlapping projections from area 4 to motor units of the baboon's hand. Proc R Soc Lond B Biol Sci 188:31-36.

Ashe J (1997) Force and the motor cortex. Behav Brain Res 86:1-15.

Beck CH, Chambers WW (1970) Speed, accuracy, and strength of forelimb movement after unilateral pyramidotomy in rhesus monkeys. J Comp Physiol Psychol 70:1-22.

Beevor C (1903a) The Croonian Lectures on muscular movements. New York: MacMillan.

Beevor C (1903b) Remarks on paralysis of the movements of the trunk in hemiplegia. New York: MacMillan.

Buys EJ, Lemon RN, Mantel GW, Muir RB (1986) Selective facilitation of different hand muscles by single corticospinal neurones in the conscious monkey. J Physiol (Lond) 381:529-549.

Chapman CE, Wiesendanger M (1982) Recovery of function following unilteral lesions of the bulbar pyramid in the monkey. Electroencepha$\operatorname{logr}$ Clin Neurophysiol 53:374-387.

Cheney PD, Fetz EE (1985) Comparable patterns of muscle facilitation 
evoked by individual corticomotoneuronal (CM) cells and by single intracortical microstimuli in primates: evidence for functional groups of CM cells. J Neurophysiol 53:786-804.

DeFelipe J, Conley M, Jones EG (1986) Long-range focal collateralization of axons arising from corticocortical cells in monkey sensory-motor cortex. J Neurosci 6:3749-3766.

Donoghue JP, Leibovic S, Sanes JN (1992) Organization of the forelimb area in squirrel monkey motor cortex: representation of digit, wrist, and elbow muscles. Exp Brain Res 89:1-19.

Evarts EV (1968) Relation of pyramidal tract activity to force exerted during voluntary movement. J Neurophysiol 31:14-27.

Fulton JF, Kennard MA (1932) A study of flaccid and spastic paralyses produced by lesions of the cerebral cortex in primates. Res Publ Assoc Res Nerv Ment Dis 13:158-210.

Georgopoulos AP, Ashe J, Smyrnis N, Taira M (1992) The motor cortex and the coding of force. Science 256:1692-1695.

Gould III HJ, Cusick CG, Pons TP, Kaas JH (1986) The relationship of corpus callosum connections to electrical stimulation maps of motor, supplementary motor, and the frontal eye fields in owl monkeys. J Comp Neurol 247:297-325.

Grafton ST, Woods RP, Mazziotta JC (1993) Within-arm somatotopy in human motor areas determined by positron emission tomography imaging of cerebral blood flow. Exp Brain Res 95:172-176.

Hamuy TP (1956) Retention and performance of "skilled movements" after cortical ablations in monkeys. Bull John Hopkins Hosp 98:417-444.

Hepp-Reymond MC (1988) Functional organization of motor cortex and its participation in voluntary movements. In: Comparative primate biology (Seklis HD, Erwin J eds), pp 501-624. New York: Alan R. Liss.

Hepp-Reymond MC, Wiesendanger M (1972) Unilateral pyramidotomy in monkeys: effect on force and speed of a conditioned precision grip. Brain Res 36:117-131.

Hepp-Reymond MC, Wiesendanger M, Brunnert A, Mackel R, Unger C, Wespi, J (1970) Effects of unilateral pyramidotomy on conditioned finger movement in monkey (Macaca irus). Brain Res 24:544.

Hepp-Reymond MC, Trouche E, Wiesendanger M (1974) Effects of unilateral and bilateral pyramidotomy on a conditioned rapid precision grip in monkeys (Macaca fascicularis). Exp Brain Res 21:519-527.

Hepp-Reymond MC, Wyss UR, Anner R (1978) Neuronal coding of static force in the primate motor cortex. J Physiol Paris 74:287-291.

Hoffman DS, Strick PL (1995) Effects of a primary motor cortex lesion on step-tracking movements of the wrist. J Neurophysiol 73:891-895.

Humphrey DR (1986) Representation of movements and muscles within the primate precentral motor cortex: historical and current perspectives. Fed Proc 45:2687-2699.

Humphrey DR, Reed DJ (1983) Separate cortical systems for control of joint movement and joint stiffness: reciprocal activation and coactivation of antagonist muscles. Adv Neurol 39:347-372.

Huntley GW, Jones EG (1991) Relationship of intrinsic connections to forelimb movement representations in monkey motor cortex: a correlative anatomic and physiological study. J Neurophysiol 66:390-413.

Jacobs KM, Donoghue JP (1991) Reshaping the cortical motor map by unmasking latent intracortical connections. Science 251:944-947.

Keller A, Asanuma H (1993) Synaptic relationships involving local axon collaterals of pyramidal neurons in the cat motor cortex. J Comp Neurol 336:229-242.

Kleinschmidt A, Nitschke MF, Frahm J (1997) Somatotopy in the human motor cortex hand area. A high-resolution functional MRI study. Eur J Neurosci 9:2178-2186.

Kubota K (1996) Motor cortical muscimol injection disrupts forelimb movement in freely moving monkeys. NeuroReport 7:2379-2384.

Kwan HC, MacKay WA, Murphy JT, Wong YC (1978) Spatial organization of precentral cortex in awake primates. II. Motor outputs. J Neurophysiol 41:1120-1131.

Laursen AM (1977) Task dependence of slowing after pyramidal lesions in monkeys. J Comp Physiol Psychol 91:897-906.

Lawrence DG, Kuypers HG (1968) The functional organization of the motor system in the monkey. I. The effects of bilateral pyramidal lesions. Brain 91:1-14.

Martin JH (1991) Autoradiographic estimation of the extent of reversible inactivation produced by microinjection of lidocaine and muscimol in the rat. Neurosci Lett 127:160-164.

Martin JH, Ghez C (1993) Differential impairments in reaching and grasping produced by local inactivation within the forelimb representation of the motor cortex in the cat. Exp Brain Res 94:429-443.

Martin JH, Cooper SE, Ghez C (1993) Differential effects of local inac- tivation within motor cortex and red nucleus on performance of an elbow task in the cat. Exp Brain Res 94:418-428.

Mewes K, Cheney PD (1991) Facilitation and suppression of wrist and digit muscles from single rubromotoneuronal cells in the awake monkey. J Neurophysiol 66:1965-1977.

Nudo RJ, Milliken GW (1996) Reorganization of movement representations in primary motor cortex following focal ischemic infarcts in adult squirrel monkeys. J Neurophysiol 75:2144-2149.

Nudo RJ, Wise BM, Sifuentes F, Milliken GW (1996) Neural substrates for the effects of rehabilitative training on motor recovery after ischemic infarct. Science 272:1791-1794.

Passingham RE, Perry VH, Wilkinson F (1983) The long-term effects of removal of sensorimotor cortex in infant and adult rhesus monkeys. Brain 106:675-705.

Penfield W, Boldrey E (1937) Somatic motor and sensory representation in the cerebral cortex of man as studied by electrical stimulation. Brain 37:389-443.

Penfield W, Rasmussen T (1950) The cerebral cortex of man. New York: MacMillan.

Porter R, Lemon RN (1993) Corticospinal function and voluntary movement. Oxford: Clarendon.

Preuss TM, Stepniewska I, Jain N, Kaas JH (1997) Multiple divisions of macaque precentral motor cortex identified with neurofilament antibody SMI-32. Brain Res 767:148-153.

Rouiller EM, Yu XH, Tempini A (1997) Effect of inactivation of the hand representation of the primary and supplementary motor cortical areas on precision grip performance in monkeys. In: Perspectives of motor behavior and its neural basis (Hepp-Reymond MC, Marini G, eds), pp 33-43. New York: Karger.

Sanes JN, Donoghue JP, Thangaraj V, Edelman RR, Warach S (1995) Shared neural substrates controlling hand movements in human motor cortex. Science 268:1775-1777.

Sato KC, Tanji J (1989) Digit-muscle responses evoked from multiple intracortical foci in monkey precentral motor cortex. J Neurophysiol 62:959-970.

Schieber MH (1990) How might the motor cortex individuate movements? Trends Neurosci 13:440-445.

Schieber MH (1991) Individuated finger movements of rhesus monkeys: a means of quantifying the independence of the digits. J Neurophysiol 65:1381-1391.

Schieber MH (1995) Muscular production of individuated finger movements: the roles of extrinsic finger muscles. J Neurosci 15:284-297.

Schieber MH, Hibbard LS (1993) How somatotopic is the motor cortex hand area? Science 261:489-492.

Schwartzman RJ (1978) A behavioral analysis of complete unilateral section of the pyramidal tract at the medullary level in Macaca mulatta. Ann Neurol 4:234-244.

Shinoda Y, Yokota J, Futami T (1981) Divergent projection of individual corticospinal axons to motoneurons of multiple muscles in the monkey. Neurosci Lett 23:7-12.

Smith AM, Hepp-Reymond MC, Wyss UR (1975) Relation of activity in precentral cortical neurons to force and rate of force change during isometric contractions of finger muscles. Exp Brain Res 23:315-332.

Soechting JF, Flanders M (1997) Flexibility and repeatability of finger movements during typing: analysis of multiple degrees of freedom. J Comp Neurosci 4:29-46.

Strick PL, Preston JB (1978) Multiple representation in the primate motor cortex. Brain Res 154:366-370.

Tower SS (1940) Pyramidal lesion in the monkey. Brain 63:36-90.

Travis AM (1955) Neurological deficiencies after ablation of the precentral motor area in Macaca mulatta. Brain 78:155-173.

Woolsey CN, Settlage PH, Meyer DR, Sencer W, Hamuy TP, Travis AM (1951) Patterns of localization in precentral and "supplementary" motor areas and their relation to the concept of a premotor area. Res Publ Assoc Res Nerv Ment Dis 30:238-264.

Woolsey CN, Gorska T, Wetzel A, Erickson TC, Earls FJ, Allman JM (1972) Complete unilateral section of the pyramidal tract at the medullary level in Macaca mulatta. Brain Res 40:119-123.

Woolsey CN, Erickson TC, Gilson WE (1979) Localization in somatic sensory and motor areas of human cerebral cortex as determined by direct recording of evoked potentials and electrical stimulation. J Neurosurg 51:476-506.

Ziemann U, Rothwell JC, Ridding MC (1996) Interaction between intracortical inhibition and facilitation in human motor cortex. J Physiol (Lond) 496:873-881. 